\title{
1 Acute cellular and molecular responses and chronic adaptations to \\ 2 low-load blood flow restriction and high-load resistance exercise in \\ 3 trained individuals
}

\section{Running head: Molecular responses to blood flow-restricted exercise}

5 Charlie J Davids*1,2, Tore C Næss ${ }^{3}$, Maria Moen ${ }^{3}$, Kristoffer T Cumming ${ }^{3}$, Oscar Horwath ${ }^{4}$,

6 Niklas Psilander ${ }^{4}$, Björn Ekblom ${ }^{4}$, Jeff S Coombes ${ }^{1}$, Jonathan Peake ${ }^{2,5}$, Truls Raastad ${ }^{3}$, and

7 Llion A Roberts*1,2,6

$8{ }^{1}$ School of Human Movement and Nutrition Sciences, The University of Queensland,

9 Brisbane, Australia; ${ }^{2}$ Queensland Academy of Sport, Nathan, Australia; ${ }^{3}$ Department of

10 Physical Performance, Norwegian School of Sport Science, Oslo, Norway; ${ }^{4}$ Åstrand

11 Laboratory, Department of Physiology, Nutrition and Biomechanics, Swedish School of

12 Sport and Health Sciences, Stockholm, Sweden; ${ }^{5}$ Queensland University of Technology,

13 School of Biomedical Science, Brisbane, Australia; ${ }^{6}$ Griffith Sports Science, School of

14 Health Sciences and Social Work, Griffith University, Gold Coast, Australia.

\section{Corresponding author:}

16 Llion Arwyn Roberts

17 Griffith Sports Science

18 School of Health Sciences and Social Work

19 Griffith University, Southport, 4215

20 Email: 1 lion.roberts@griffith.edu.au

21 Tel: +61(0) 755528451

22 Fax: $++61(0) 755528674$

23 ORCID ID: 0000-0002-2284-1033 
25 Blood flow restriction (BFR) with low-load resistance exercise (RE) is often used as a surrogate to traditional high-load RE to stimulate muscular adaptations, such as hypertrophy and strength. However, it is not clear whether such adaptations are achieved through similar cellular and molecular processes. We compared changes in muscle function, morphology and signaling pathways between these differing training protocols. Twenty-one males and females (mean \pm SD: $24.3 \pm 3.1$ years) experienced with resistance training $(4.9 \pm 2.6$ years) performed nine weeks of resistance training (three times per week) with either high-loads (75-80\% 1RM; HL-RT), or low-loads with BFR (30-40\% 1RM; LL-BFR). Before and after the training intervention, resting muscle biopsies were collected, and quadricep crosssectional area (CSA), muscular strength and power were measured. Approximately 5 days following the intervention, the same individuals performed an additional 'acute' exercise session under the same conditions, and serial muscle biopsies were collected to assess hypertrophic- and ribosomal-based signaling stimuli. Quadricep CSA increased with both LL-BFR $(7.4 \pm 4.3 \%)$ and HL-RT $(4.6 \pm 2.9 \%)$, with no significant differences between training groups $(p=0.37)$. Muscular strength also increased in both training groups, but with superior gains in squat $1 \mathrm{RM}$ occurring with HL-RT $(p<0.01)$. Acute phosphorylation of several key proteins involved in hypertrophy signaling pathways, and expression of ribosomal RNA transcription factors occurred to a similar degree with LL-BFR and HL-RT (all $p>0.05$ for between-group comparisons). Together, these findings validate low-load resistance training with continuous BFR as an effective alternative to traditional high-load resistance training for increasing muscle hypertrophy in trained individuals. 


\section{NEW \& NOTEWORTHY}

50 Low-load resistance exercise with blood flow restriction (LL-BFR) is an effective

51 method for stimulating muscular adaptations, but phenotypical and mechanistic comparisons

52 with traditional high-load training (HL-RT) in trained populations are scarce. The findings

53 indicate that hypertrophy, but not strength, is comparable between LL-BFR and HL-RT, and

54 the acute cellular and molecular processes for hypertrophy were similar, but not identical,

55 between protocols. Thus, LL-BFR is an effective alternative to HL-RT for obtaining

56 hypertrophy in trained populations.

57 Key Words: OCCLUSION, ISCHEMIA, HYPOXIA, KAATSU, HYPERTROPHY 
It is generally recommended that individuals perform resistance training with external loads of at least $70 \%$ of their one-repetition maximum (1RM) to maximize the positive outcomes of resistance training (e.g. muscle hypertrophy and increase strength) (1). Such loading conditions place high levels of mechanical tension on muscle tissue, which is believed to act as a primary mediator of intracellular anabolic signaling pathways, and consequently hypertrophic adaptations via mechanotransduction (2). However, in certain situations, it is often necessary to limit mechanical loading while facilitating muscular development; for example, when periodizing an athlete's total training stress (3), or during rehabilitation of musculoskeletal injury (4). exhibits a hypertrophic response to both mechanical and metabolic stimuli $(5,6)$.

Specifically, metabolic by-products induced by a high volume of muscle contractions, limited

71 rest durations, or restricting blood flow (BFR) to the exercising muscles appear to

72 compensate for reduced mechanical loading and achieve similar outcomes in skeletal muscle

$73(5,7,8)$. Indeed, much lower loads e.g., 20-50\% of 1RM with BFR (LL-BFR) have been

74 used to elicit similar chronic hypertrophy to high-load non-restricted exercise (9-12), and

75 similar rates of myofibrillar protein synthesis (13). This supports the belief that muscle fiber

76 recruitment and the downstream anabolic processes responsible for hypertrophy may be

77 achieved through means other than high loading conditions $(6,7,14)$. Consequently, LL-BFR

78 has been celebrated as a promising strategy to deliver muscular benefits to those populations

79 who desire periodized exposure to high mechanical loads, or cannot tolerate such loads (4, $80 \quad 15)$.

81 Importantly, little is known regarding how LL-BFR compares with traditional high-

82 load training ( $>70 \%$ 1RM, HL-RT), particularly at the intramuscular level. Although the 
magnitude of hypertrophy achieved with training appears to be similar between these protocols (12), there is conflicting evidence on how such differences in loading conditions influence morphological adaptations at the fiber level $(10,13,16,17)$. There is some evidence suggesting that BFR provides a greater metabolic stimulus, with low loads preferentially stressing type I muscle fibers $(18,19)$. Some chronic studies align with these acute findings and report greater increases in type I fiber area with LL-BFR training $(16,17)$. Together these data suggest that type I fibers may be selectively targeted when using low loads with BFR.

It is crucial to consider whether such fiber-type specific adaptations influence characteristics of muscular performance. For example, if BFR with low loads targets type I fibers at the expense of type II fibers, this may result in inferior strength and especially power adaptations. Such outcomes would be highly undesirable for some athletic cohorts after resistance training (20). Although athletes are unlikely to use LL-BFR training exclusively in the long term, it is necessary to gauge whether this training mode has a comparable influence on the skeletal muscle phenotype compared with traditional HL-RT. This comparison of resistance training protocols will also help inform practitioners and coaches about how best to structure each training mode—and therefore the proportion of mechanical and metabolic stimuli — to develop the desired physical qualities for differing individuals and sports. Many of the cellular mechanisms underpinning BFR exercise remain unclear. Following traditional resistance exercise, it is well recognized that key intracellular signaling pathways such as mammalian target of rapamycin (mTOR) and mitogen-activated protein kinase (MAPK) are activated (21-23) to aid in the muscle remodeling process. More recently, the importance of ribosomal biogenesis to this process has also come to light (24) for its role in increasing ribosomal capacity (25) and subsequently contributing to muscle hypertrophy as a result of a greater translational ability. Indeed, both the transcription of rRNA genes (26) 
and mTOR pathway activity through the ribosomal protein S6 kinase (27) are strongly correlated with muscle hypertrophy. However, it is not clear if these key cellular and molecular responses occur in the same fashion following LL-BFR exercise, and if so, how these responses compare in magnitude to traditional HL-RT. If divergent mechanistic pathways to muscle hypertrophy exist between protocols, this may suggest there are synergistic possibilities for muscular adaptations if both approaches are included within a program (6).

The aims of this study were to (i) compare how LL-BFR and HL-RT influence chronic morphological, molecular and functional responses in trained individuals, and (ii) examine acute cell signaling and gene expression responses associated with muscle hypertrophy following resistance exercise. It was hypothesized that (i) skeletal muscle crosssectional area (CSA) would increase similarly between conditions following the training period, which would be corroborated by similar acute signaling and gene expression responses, and (ii) that despite similar increases in muscle CSA, LL-BFR would preferentially increase the size of type I fibers, whereas HL-RT would preferentially increase the size of type II fibers.

\section{METHODS}

An overview of the methods are detailed herein. Additional detail pertaining to the subsections can be found within the supplementary methods file specified in the endnote.

Experimental Design. The present study involved two chronologically sequential experimental parts, investigating firstly the chronic, and then secondly the acute responses to two resistance exercise protocols. The first part consisted of a randomized controlled trial in which participants performed 9 weeks of lower body strength training. Prior to the training 
133 period, participants were stratified and block randomized into two groups based on absolute

134 lean lower body mass (using dual energy x-ray absorptiometry), relative squat strength, and

135 sex. Subsequently, the groups were randomly assigned to become a high-load resistance

136 training group (HL-RT; $n=10)$, or a low-load resistance training with blood flow restriction

137 group (LL-BFR; $n=11)$. Muscle mass and strength were assessed, and resting muscle

138 biopsies were collected from $m$. vastus lateralis before and within 5-7 days after training.

139 Following 4-5 days of rest after the completion of post-training assessments, the same

140 participants returned to complete the second part of the study, which was an acute exercise

141 trial. Muscle biopsies and performance-based assessments were obtained following a single

142 exercise session that was representative of the sessions performed in the chronic training

143 study. The post-training measures after the 9 weeks of training represented the baseline

144 measures for the acute study. All experimental procedures adhered to the standards set by the

145 Declaration of Helsinki and were approved by the ethical committees of the Norwegian

146 School of Sport Science and the Norwegian Center for Research Data (protocol number 63-

147 190618).

149 Subjects. Twenty-four healthy males and females were recruited to participate in the study.

150 Sample size was determined from previous literature adopting similar experimental designs

151 and outcome measures as those included in the present study (10, 28, 29). All participants

152 performed regular resistance training for a minimum of 2 years leading up to the study

153 (training history $4.9 \pm 2.6$ years; relative squat strength $1.7 \pm 0.2$ times body mass for males,

$1541.1 \pm 0.2$ times body mass for females). Before providing their written informed consent, the

155 requirements and risks involved in the experiments were explained to participants, and

156 participants were screened using the Australian Adult Pre-exercise Screening System (APSS)

157 (30). Participants were considered eligible to participate if they had consistently performed 
158 lower body resistance training at least once per week for the last 2 years, and were familiar

159 with the barbell back squat exercise. Further exclusion criteria included cardiovascular

160 disease, musculoskeletal injury, pregnancy, or those taking medications known to enhance

161 blood clotting risk. 21 participants completed the training study and the associated

162 assessments, after 3 participants withdrew citing personal reasons unrelated to the study. Pre-

163 and post- training muscle biopsies were not obtained from one participant in LL-BFR due to

164 personal preferences, however all other measures for this individual were completed. Another

165 participant from LL-BFR was excluded from immunohistochemical analyses because the

166 quality of their biopsy tissue was not adequate for analysis. For the acute study, two

167 participants in HL-RT and one participant in LL-BFR opted not to participate due to

168 availability or personal preferences. All acute measures and analyses were completed for the

169 remaining participants (HL-RT: $n=8$; LL-BFR: $n=10$ ). The participant characteristics for

170 each study component are outlined in Table 1.

171

172 Training Study

173 Resistance Training. An overview of the resistance training program is displayed in Table 2.

174 The 9-week training block consisted of three supervised lower-body resistance sessions per

175 week, for a total of 26 sessions. Exercise volume and frequency were reduced during week 5

176 of training to promote recovery and adaptation (3). Resistance training was progressive,

177 including barbell back squat, leg press, knee extension and Bulgarian split squat exercises.

178 This combination of multi-joint and single-joint exercises was selected to provide our

179 previously strength-trained participants with sufficient volume to promote training

180 adaptations, and to reflect more the typical lower body resistance exercises adopted in athletic

181 programs. Loads were adjusted on a session-by-session basis, using repetitions in reserve

182 (RIR) to gauge intensity, and to attempt to standardise the proximity to muscular failure 
between participants and training groups. Loads were progressed if RIR for consecutive sets exceeded two repetitions in HL-RT, and four repetitions in LL-BFR. Conversely, if the desired number of repetitions could not be completed, or muscular failure occurred during exercise, loads were reduced for the subsequent session. This method of progressive overload was chosen primarily to serve as a more ecologically valid comparison of HL-RT and LLBFR training protocols, and to eliminate the inherent bias that occurs when attempting to match volume between low-load and high-load conditions (31). In addition, it allowed for investigation of the external loads that trained individuals can tolerate with BFR, and consequently, how much volume each group would perform.

Blood Flow Restriction. In the LL-BFR group, individualized BFR cuff pressures were determined using Doppler ultrasound (Phillips, HD15 PureWave). While seated in the upright position, the posterior tibial artery was imaged while a 10-cm nylon cuff (Sports Rehab Tourniquet, Brisbane, AU) was positioned around the most proximal region of the dominant thigh, and inflated incrementally. Arterial occlusion pressure (AOP) was recorded as the minimum pressure at which arterial pulse waves were no longer detected visually or aurally. During training, the same nylon cuffs were positioned around the most proximal region of both thighs. The cuffs were then inflated manually using a handheld sphygmomanometer to a pressure corresponding to $60 \%$ AOP. Cuffs were inflated immediately before the first set of each exercise, and remained inflated during the inter-set recovery periods. Following the final set of each exercise, the cuffs were deflated. Between exercises, cuffs remained positioned around the thighs, but were not inflated.

Assessments of Muscle Function. Muscular strength and power were measured four times over the course of the study. Prior to training, the series of assessments were performed 
twice, each separated by a week. The first occasion was to familiarize participants with the testing protocols, before collecting the true baseline measures the following week. Midway through the training period, muscular strength tests were repeated. Following the training intervention, all muscle function assessments were performed between 5-7 days following the final training session. The details of each assessment involved are described below. Isometric strength of the dominant knee extensors and flexors was assessed during unilateral maximal isometric contractions, using a dynamometer (HUMAC Norm, CSMi, Stoughton, MA) as previously described (28).Participants performed three 3-second maximal voluntary contractions (MVCs) of the knee extensors, each separated by 120 seconds. Subsequently, three MVCs of the knee flexors were performed at the same knee angle. For all MVCs, participants were instructed to apply force maximally and as rapidly as possible for the entire 3 secondsTest-retest CV values were $4.7 \%$ and $4.2 \%$ for peak torque during isometric knee extension and knee flexion contractions, respectively.Barbell back squat onerepetition maximum (1RM) was measured to assess dynamic strength, and to establish initial training loads during the studyFailure to complete the concentric (raising) portion of the movement, or not achieving the specified range of motion (90-degree knee angle); were deemed as an unsuccessful attempt. The highest load that was successfully completed was recorded as the 1RM score. Finally, countermovement and squat jumps were performed on a force plate (FP4, HUR Labs, Kokkola, Finland) to assess lower body power and velocity characteristics. Participants were then instructed to jump for maximal height, for three attempts for each style of jump.Test-retest CV values were $5.7 \%$ and $5.4 \%$ for jump height by takeoff velocity for countermovement and squat jumps, respectively. Test-retest CV values were $4.0 \%$ and $4.3 \%$ for peak power for countermovement and squat jumps, respectively. 
Assessments of Muscle Mass. Muscle mass was quantified using two methods. First,

the summated CSA of the quadriceps muscle group of the dominant leg was measured using magnetic resonance imaging (MRI; Magnetom Avanto 1.5T, Siemens AG, Munich,

Germany). Second, lean muscle mass was measured using Dual Energy X-ray

Absorptiometry (DEXA; Lunar iDXA, GE Healthcare, Buckinghamshire, UK). MRI scans were performed over days 12 and 13 before commencing training, and again 5 days following the final training session, before the post-training biopsy and acute component of the study commenced. Participants were instructed to abstain from any exercise in the 24 hours before each scan. Fifteen separate transverse section images were collected; these images were equally distributed relative to the participant's femur length between the greater trochanter and the lateral epicondyle. From these 15 images, images three to 12 ( 1 being most distal) were selected for each participant for analysis. Images one and two, and 13-15 were not used due to poor image contrast owing to placement of the coil. The 10 images were analyzed using ITK-SNAP software (University of PA, Philadelphia, USA; www.itksnap.org) to generate individual CSAs, which were then summated. The test-retest CV for total quadricep CSA from six participants' scans was $1.6 \%$. Whole body DEXA imaging was conducted on participants in a fasted but hydrated state, after abstaining from exercise in the previous 24 hours. Lower body lean mass was determined from each scan to firstly assist with block randomization participants at baseline, as well as providing a secondary measure of muscle mass changes following training. The test-retest $\mathrm{CV}$ for lower body lean mass from DEXA was $1.7 \%$.

Collection of Muscle Tissue. Resting muscle biopsies were obtained from the mid-portion of m. vastus lateralis from the dominant leg of each participant. Baseline biopsies were collected 9-10 days before the first training session. Post-training biopsies were collected 
5-7 days after the final training session, to align with the acute component of the study. Posttraining biopsies were collected $\sim 3 \mathrm{~cm}$ proximal to the pre-training site. All muscle biopsies were collected under local anesthesia $\left(10 \mathrm{mg} \cdot \mathrm{ml}^{-1}\right.$ xylocaine $+5 \mu 1 \cdot \mathrm{ml}^{-1}$ adrenaline,

260 AstraZeneca, London, UK) with a sterile 6-mm Bergstrom needle (Pelomi, Albertslund,

261 Denmark). Following collection, the biopsy sample was quickly cleaned for visual fat and connective tissue. Samples intended for immunohistochemistry were aligned and covered in O.C.T embedding matrix (CellPath, Newtown, UK) and frozen in dry ice cooled isopentane. Samples for western blotting were gently cleaned for non-muscle tissue in cold physiological saline before being snap frozen in liquid nitrogen. All samples were transferred to storage at $-80^{\circ} \mathrm{C}$ for subsequent analysis. Muscle tissue intended for RT-PCR was immersed in RNAlater ${ }^{\circledR}$ solution (Ambion, TX, USA), stored for $24-48$ hours at $4{ }^{\circ} \mathrm{C}$, and thereafter stored at $-20{ }^{\circ} \mathrm{C}$ for later treatment and analysis.

Assessment of Muscle Fiber Type, Fiber Area, and Myonuclear Number. Mean CSA analysis included 269 type I fibers (range, 115-590) and 408 type II fibers (range, 1041024). Nuclei positive for DAPI and PCM1 were counted and quantified as myonuclei and related to respective fiber types. A random selection of 50 type I and 50 type II fibers were included in the analysis. See supplementary file for additional detail.

Control Procedures. Before baseline and post-training testing sessions, participants were instructed to refrain from consuming any stimulants or ergogenic aids that may influence performance. During the 9 weeks of training, $24 \mathrm{~h}$ food diaries were collected each week, including a mixture of training days, non-training days and weekend days. General recommendations were made to participants to consume a diet rich in protein (e.g. 
282 following each training session (100\% Whey, ProteinFabrikken, Norway). Participants were 283 also instructed to refrain from taking any performance supplements (e.g., creatine) during the

284 study, as well as to refrain from any additional lower body strength training. Upper body

285 resistance training and endurance training were permitted, and were recorded weekly in an

286 exercise diary. Participants were also asked to limit high-intensity exercise (e.g., intervals-

287 based training) to twice weekly at most, details of which were also diarized.

288 For all outcome measures, assessors were blinded to group assignment where

289 possible. For laboratory and imaging assessments (e.g. Western blotting, magnetic resonance

290 imaging etc.) assessors were blinded to group assignment. All baseline performance

291 assessments (e.g. 1RM, isometric strength etc.) were collected prior to group assignment.

292 Post-training performance assessments were not blinded due to financial constraints.

Adverse events. During the training study, one participant in the LL-BFR group reported an exercise-induced migraine which would return following any physical exertion. Following a two-week break from training, the participant could complete sessions free of pain. The participant completed 25 out of 26 sessions in the training block.

298

299 Acute Study

300 Experimental Design. The second experiment in the study involved performing an acute training session to evaluate the short-term molecular signaling and muscle function responses to each training approach. This was initiated approximately 5-7 days following the final

303 training session, and $\sim 3-5$ days after post-training assessments were completed, where participants returned to complete a single lower body resistance exercise session. Then at 2 , 305 24 and 48 hours after exercise, muscle biopsies were collected, and muscle function was 
assessed to evaluate fatigue and subsequent recovery. The post-training measures were used as the pre-exercise time point here.

Acute Exercise Session. The exercise session completed in the acute study was the same as that in the training study (outlined in Table 2). Participants remained in the same groups to which they were previously allocated; therefore, those individuals who had previously trained for 9 weeks using BFR, performed the session with BFR, and vice versa. The exercise loads used in the acute session were based on those used in the final training session, and were adjusted if necessary, to maintain 1-2 RIR and 2-4 RIR for HL-RT and LL-BFR, respectively. The RIR method was selected over other conventional methods (e.g. \%1RM), to mirror the stimulus provided by each session of the subsequent training period, and to standardize perceived effort and proximity to failure across participants and exercise protocols. Acute responses were investigated at the end of the training block to minimize any exaggerated responses that occur when participants are unaccustomed to a particular training protocol.

Collection of Muscle Tissue. The process for obtaining and processing muscle biopsies in the acute study was the same as that previously described for the training study, aside from the details below. The $2 \mathrm{~h}$ post-exercise biopsy was collected through the same incision as the post-training/pre-exercise biopsy, but with alternate needle direction to ensure $\sim 4 \mathrm{~cm}$ distance between biopsy collection sites. Biopsies obtained at 24 and $48 \mathrm{~h}$ post-exercise were taken from the non-dominant leg, from separate incisions, to reduce the number of samples taken from one muscle. Two hours preceding each biopsy (with the exception of the $2 \mathrm{~h}$ postexercise biopsy), a standardized oatmeal meal ( $0.16 \mathrm{~g}$ protein $\cdot \mathrm{kg}^{-1}$ body mass) was provided to participants. A serving of whey protein isolate $\left(0.4 \mathrm{~g}\right.$ protein $\cdot \mathrm{kg}^{-1}$ body mass $)$ was provided 
331

332

333

334

335

336

337

338

339

340

341

342

343

344

345

346

347

348

349

350

351

352

353

354

355

to participants upon completing the acute exercise session (i.e. two hours prior to the $2 \mathrm{~h}$ post-exercise biopsy).

Western Blotting. Please see supplementary methods file.

RNA extraction and cDNA synthesis.. Please see supplementary methods file.

Real-Time qPCR. Please see supplementary methods file.

Assessments of Muscle Function. Two performance tests were completed before, and after exercise to determine acute decrements in muscle function, as measures of the fatigue and recovery timelines for each exercise type. Performance assessments were completed following the collection of the pre-exercise biopsy, approximately 5 minutes post-exercise following ingestion of whey protein isolate, and following the 2, 24 and $48 \mathrm{~h}$ biopsy collections. Unilateral isometric peak torque was first measured from the non-dominant knee extensors, as described above in the training study methodology. Subsequently, three maximal countermovement jumps were performed (also as detailed previously in the training study methodology) to evaluate lower body power and velocity characteristics.

Assessments of Thigh Volume and Soreness. At the same time points as the muscle function assessments, the circumference of the non-dominant thigh of the participants was measured. They were subsequently used to estimate total thigh volume using the formula for segmental limb volume developed by Katch and Katch (32). Participants were also asked to rate their perceptions of soreness (CR-10+) while performing an isometric squat at 90 degrees, at each time-point. 
357 Statistical Analyses. Statistical analyses were performed using the Statistical Package for

358 Social Sciences (SPSS; v24, IBM, Armonk, NY, USA). Data were firstly checked for

359 normality using the Shapiro-Wilk test. Protein phosphorylation data was log transformed to

360 conform to normal distribution. Two-factor analysis of variance (ANOVA) with repeated-

361 measures were performed to assess the effects of group (LL-BFR vs HL-RT) and time (PRE

362 vs POST training) for the training study. For the acute study, two-factor ANOVA with

363 repeated-measures were also performed to assess the effects of group (LL-BFR vs HL-RT)

364 and time (pre, 0, 2, 24, $48 \mathrm{~h}$ post). Any significant main effects were followed with post-hoc

365 Student's $t$ tests (unpaired for significant group interactions and paired for significant time

366 effects) to identify the origin of the significant difference. To account for multiplicity, the

367 False Discovery Rate (FDR) method of Benjamini and Hochberg (33) was selected instead of

368 Bonferroni's correction, to reduce the possibility of type II error (given the limited sample

369 size, and small number of pair-wise comparisons made in the present study (34)). Effect sizes

$370(d)$ for paired data (e.g. pre vs post) were calculated based on the identified mean differences

371 and the standard deviation (SD) of these differences (35). For unpaired data (e.g. LL-BFR vs

372 HL-RT), traditional Cohen's $d$ effect sizes were computed using pooled SD. All effect sizes

373 were interpreted as; $d<0.2=$ null effect, $d<0.5=$ small effect, $d<0.8=$ moderate effect, and

$374 d>0.8=$ a large effect (36). Data are presented as mean \pm SD with $95 \%$ confidence intervals

375 (CI) unless stated otherwise. Statistical significance was accepted as $p \leq 0.05$.

376 


\section{RESULTS}

\section{Training Study}

379 Adherence. All 21 participants completed the training study with high adherence. To be included in the final analyses, participants were required to complete at least 24 sessions during the training block. Participants undertaking LL-BFR completed $25.5 \pm 0.8$, and HLRT completed $25.7 \pm 0.5$, out of 26 sessions, respectively.

No meaningful differences were observed between groups when analyzing participants' diet and exercise diaries across the training period.

Training Load Progression. The average volume-loads (sets $\times$ reps $\times$ load) performed during squat, leg press and leg extension exercises were calculated during the first and final weeks of training to evaluate load progressions between protocols. There was a time*group interaction $(p=0.004)$ and time effect $(p<0.001)$ for volume-load. Volume-load increased with HL-RT from $7,796 \pm 3,027 \mathrm{~kg}$ to $10,279 \pm 2,969 \mathrm{~kg}(p=0.001 ; \sim 38 \%)$, and in LL-BFR from 7,179 \pm $3,463 \mathrm{~kg}$ to $12,317 \pm 4,480 \mathrm{~kg}(p<0.001 ; \sim 79 \%)$. The volume-load increase was greater in LL-BFR $(5,138 \pm 2,020 \mathrm{~kg}[95 \% \mathrm{CI}: 3,781$ to 6,494$])$ than in HL-RT $(2,483 \pm 1502 \mathrm{~kg}[95 \%$ CI: 1,328 to 3,637$], p=0.004)$. For example, HL-RT participants began training the back squat with a load of $73.7 \pm 2.7 \%$ of baseline $1 \mathrm{RM}$, and finished training with a load of $91.4 \pm$ $11.8 \%$ of baseline 1RM. By contrast, LL-BFR participants began training with a load of 30.5 $\pm 3.3 \%$ of baseline $1 \mathrm{RM}$, and finished training with a load of $56.3 \pm 9.8 \%$ of baseline 1RM.

Changes in Muscle Morphology. Changes in muscle morphology are displayed in Figure 1.

399 There were no time*group interactions for summated cross-sectional area (CSA; Figure 1A)

400 of the quadriceps $(p=0.369)$, nor DEXA fat-free mass (Figure 1B) $(p=0.185)$. However, a 
401 time effect $(p<0.001)$ was evident. Quadriceps CSA increased after HL-RT $\left(92.7 \pm 22.1 \mathrm{~cm}^{2}\right.$

402 [95\% CI: 36.4 to 149.0$], d=1.52)$ and LL-BFR $\left(118.8 \pm 17.6 \mathrm{~cm}^{2}\right.$ [95\% CI: 80.7 to 156.9 ],

$403 d=2.09$ ). Lower body lean mass increased after HL-RT (194 $\pm 98 \mathrm{~g}$ [95\% CI: 9 to 379],

404

$d=0.75)$ and LL-BFR (380 $\pm 93 \mathrm{~g}$ [95\% CI: 146 to 614$], d=1.09)$.

405

Muscle fiber CSA and myonuclei number changes are displayed in Figure 2. No

406

time*group interactions existed for type I fiber CSA $(p=0.331)$ and myonuclei number

407

$(p=0.139)$ or type II fiber CSA $(p=0.677)$ and myonuclei number $(p=0.113)$. However, a time

408

effect existed for type II fiber CSA $(p=0.010)$ and myonuclei accretion $(p=0.029)$, but not

409

type-I fibers (both $p>0.05)$. Type II fiber CSA increased after HL-RT $\left(998 \pm 416 \mu \mathrm{m}^{2},[95 \%\right.$

410 CI: 700 to 1296$], d=0.55)$, and after LL-BFR (742 $\pm 438 \mu \mathrm{m}^{2}$ [95\% CI: 405 to 1079],

411

$d=0.66)$. Myonuclei accretion increased after HL-RT for both type I $(17 \pm 23 \%$ [95\% CI: 0 to

$41234], d=0.73)$ and type II fibers ( $24 \pm 31 \%$ [95\% CI: 2 to 46$], d=0.77)$, whereas no change

413 was observed after LL-BFR for type I fibers ( $3 \pm 21 \%$ [95\% CI: -13 to 19$], d=0.05)$ or type

414 II fibers $(5 \pm 15 \%$ [95\% CI: -7 to 16$], d=0.23)$.

415

416 Changes in Muscle Function. Changes in muscle function, confidence intervals and effect

417 sizes are reported in Table 4. There was no time* group interaction $(p=0.508)$ for maximal

418 knee extensor isometric torque; however, a time effect was observed $(p=0.003)$. Torque

419 increased in HL-RT $(9.7 \%, p=0.031)$, but not in LL-BFR $(5.2 \%, p=0.253)$. Knee flexor

420 torque showed a trend toward a time* group interaction $(p=0.059)$, but a time effect was

421 present $(p=0.050)$. Maximal flexion torque increased in LL-BFR $(15.0 \%, p=0.016)$, but not

422 in $\operatorname{HL}-\mathrm{RT}(1.9 \%, p=0.951)$.

423 A time*group interaction $(p=0.008)$ and time effect $(p<0.001)$ was evident for back

424 squat 1RM. Squat strength increased both in HL-RT $(17.5 \%, p<0.001)$ and LL-BFR $(9.3 \%$,

$425 p<0.001)$. The increase was greater in HL-RT than in LL-BFR $(p=0.024, d=1.02)$. 
No time*group interaction $(p=0.058)$ or time effect $(p=0.092)$ existed for squat jump

427 (SJ) height. However, the data suggested a strong tendency for SJ height to increase with HL-

428 RT $(+7.2 \%, 2.3 \mathrm{~cm}[95 \%$ CI: 0.4 to 4.1$], d=0.89)$, but not with LL-BFR $(0.1 \%,-0.1 \mathrm{~cm}$

429 [95\% CI: -1.9 to 1.6$], d=0.0)$. There were no time effects or time*group interactions for

430 counter-movement jump (CMJ) height or peak power (all $p>0.05)$.

431

432 Acute Study

433 Anabolic Signaling. The acute protein signaling responses are displayed in Figure 3. There

434 was no time* group interaction $(p=0.088)$ for $\mathrm{p} 70 \mathrm{~S} 6 \mathrm{k}^{\mathrm{Thr} 389}$ phosphorylation, however a time

435 effect was observed $(p<0.001) \cdot \mathrm{p} 70 \mathrm{~S} 6 \mathrm{k}^{\mathrm{Thr} 389}$ phosphorylation was greater at $2 \mathrm{~h}(33.0$-fold

436 change [95\% CI: 2.3 to 63.7$], p<0.001, d=1.44), 24 \mathrm{~h}(15.6$-fold change [95\% CI: -7.3 to

$43738.6], p<0.001, d=1.20)$ and 48 h (8.6-fold change [95\% CI: 2.9 to 14.3 ] $, p<0.001, d=1.24)$

438 compared with pre-exercise after HL-RT. Following LL-BFR p70S6k ${ }^{\text {Thr389 }}$ phosphorylation

439 was greater at $2 \mathrm{~h}(9.7$-fold change [95\% CI: 0.1 to 19.3$], p<0.001, d=1.60)$ compared with

440 pre-exercise. p70S6k ${ }^{\text {Thr389 }}$ phosphorylation may have been greater 24 to $48 \mathrm{~h}$ post-exercise

441 after HL-RT compared with LL-BFR $(d=0.8)$. p70S6 $\mathrm{k}^{\text {Thr389 }}$ phosphorylation area under the

442 curve (AUC) from 2-48 $\mathrm{h}$ tended to be higher with HL-RT (825 \pm 1017 A.U.) compared with

443 LL-BFR $(179 \pm 177$ A.U, $p=0.064, d=0.89)$.

444 No time*group interaction $(p=0.450)$ or time effect existed $(p=0.186)$ for

$445 \quad \mathrm{rpS6} 6^{\mathrm{Ser} 235 / 236}$ phosphorylation.

446 No time*group interaction existed for 4EBP-1 ${ }^{\text {Thr37/46 }}$ phosphorylation $(p=0.833)$,

447 however a time effect was observed $(p<0.001) .4 \mathrm{EBP}-1^{\text {Thr37/46 }}$ phosphorylation decreased $2 \mathrm{~h}$

448 following HL-RT ( -0.6 -fold change [95\% CI: -0.8 to -0.4$], p=0.008)$ and LL-BFR $(-0.5-$

449 fold change [95\% CI: -08 to -0.3 ], $p<0.001$ ), while it returned to pre-exercise levels in both

450 groups at $24 \mathrm{~h}$ and $48 \mathrm{~h}($ all $p=0.728)$. 
No time*group interaction $(p=0.383)$ existed for ERK1/2 ${ }^{\text {Thr202/Tyr204 }}$ phosphorylation,

452 however a time effect was observed $(p=0.003)$. ERK1/2 $2^{\text {Thr202/Tyr204 }}$ phosphorylation was

453 elevated at $2 \mathrm{~h}(1.7$-fold change [95\% CI: 1.3 to 2.1 ], $p=0.004, d=1.22)$ and $48 \mathrm{~h}(2.0$-fold

454 change [95\% CI: 1.2 to 2.8$], p<0.001, d=1.12$ ) following LL-BFR. However, no increases

455 were observed following HL-RT.

456 mRNA and pre-rRNA Expression. Gene expression of transcriptional factors and pre-

457 ribosomal RNA (rRNA) are illustrated in Figure 4. No time*group interaction or time effect

$458(p=0.562)$ existed for RNA Polymerase I Subunit A (POLR1A) mRNA expression

459 ( $p=0.687)$. However, a significant group difference was observed; POLR1A mRNA was

460 greater in LL-BFR than in HL-RT, $p=0.044)$. No time* group interaction existed for c-Myc

461 mRNA expression $(p=0.606)$, however a time effect was observed $(p=0.001)$. c-Myc mRNA

462 expression increased at $2 \mathrm{~h}(32.1$-fold change [95\% CI: 16.9 to 47.4$] p<0.001), 24 \mathrm{~h}(2.2$-fold

463 change [95\% CI:1.3 to 3.0] $p<0.001)$, and $48 \mathrm{~h}(2.5$-fold change [95\% CI: 1.1 to 3.8 ]

$464 p<0.001)$ following LL-BFR. Following HL-RT, c-Myc mRNA expression increased at $2 \mathrm{~h}$

465 (27.5-fold change [95\% CI: 23.8 to $31.2, p=0.010)$ and $24 \mathrm{~h}(3.0$-fold change [95\% CI: 2.1 to

$4664.0, p=0.003)$, but not $48 \mathrm{~h}$ post-exercise (3.9-fold change [95\% CI: 0.1 to 7.7$], p=0.124)$. No

467 time* group interaction existed for transcription initiation factor 1A (TIF-1A) mRNA

468 expression $(p=0.606)$, however a time effect was observed $(p=0.002)$. TIF-1A mRNA

469 expression increased only at $48 \mathrm{~h}$ post-exercise for both LL-BFR (1.5-fold change [95\% CI:

4700.9 to 2.1$], p=0.042)$ and HL-RT (2.1-fold change [95\% CI: 1.3 to 2.9$], p=0.009)$. No

471 time* group interaction existed for TATA box-binding protein-associated factor 1A (TAF-

472 1A) mRNA expression $(p=0.830)$, however a time effect was observed $(p<0.001)$. TAF-1A

473 mRNA expression increased at $24 \mathrm{~h}$ (1.6-fold change [95\% CI: 1.1 to 2.1 ], $p=0.010)$ and $48 \mathrm{~h}$

474 (1.8-fold change [95\% CI: 1.3 to 2.4$], p=0.009)$ post-exercise for LL-BFR. Following HL-

475 RT, TAF-1A mRNA expression increased only at $24 \mathrm{~h}$ post-exercise $(2.0$-fold change [ $95 \%$ 
476 CI: 1.3 to 2.7$], p=0.031)$. No time* group interaction existed for upstream binding

477 transcription factor (UBF) mRNA expression $(p=0.970)$. UBF mRNA expression did not

478 increase following exercise $(p=0.086)$, and there were no differences between groups

479 ( $p=0.498) .45 \mathrm{~S}$ pre-rRNA expression did not increase following exercise in either group

$480(p=0.711)$. However, a significant group difference was observed (greater in LL-BFR than in

481 HL-RT, $p=0.016)$.

482

483

Total RNA Content and rRNA Transcript Expression. Total RNA content and expression of

rRNA transcripts are illustrated in Figure 5. No effects of time, group or time*group

485

interactions were observed for total muscle RNA content, 5.8S rRNA, 18S rRNA, 28S rRNA

486

or $45 \mathrm{~S}$ pre-rRNA (all $p>0.05$ ).

487

Muscle Function. Acute changes in muscle function are displayed in Figure 6. There was no time* group interaction $(p=0.866)$ for knee extensor maximal isometric torque, however a 490 time effect existed $(p<0.001)$. Knee extensor torque decreased from pre- to post-exercise after HL-RT $(-27 \pm 16 \mathrm{Nm}[95 \% \mathrm{CI}:-40$ to -13$],-12 \%, p=0.005)$ and LL-BFR $(-27 \pm 30$ $\mathrm{Nm}[95 \% \mathrm{CI}:-52$ to -2$],-12 \%, p<0.001)$. At $2 \mathrm{~h}$ post-exercise, torque remained reduced in both HL-RT $(-18 \pm 9 \mathrm{Nm}[95 \% \mathrm{CI}:-26$ to -11$],-9 \%, p=0.045)$ and LL-BFR $(-20 \pm 31 \mathrm{Nm}$ [95\% CI: -42 to 2$],-7 \%, p=0.005)$. Torque recovered to pre-exercise levels in both groups

495

496

497

498

499

500
24 to $48 \mathrm{~h}$ post-exercise (all $p>0.05$ ).

No time*group interaction ( $p=0.933)$ existed for maximal jump height achieved during the countermovement jump, however a significant time effect was observed $(p<0.001)$. Jump height was reduced from pre- to post-exercise after HL-RT $(-5 \pm 4 \mathrm{~cm}$ [95\% CI: -9 to -2$],-11 \%, p<0.001)$ and LL-BFR $(-4 \pm 3 \mathrm{~cm}[95 \% \mathrm{CI}:-6$ to -2$],-12 \%$, $p<0.001)$. At $2 \mathrm{~h}$ post-exercise, jump height remained reduced in both HL-RT $(-2 \pm 1 \mathrm{~cm}$ 
$501[95 \% \mathrm{CI}:-3$ to -1$],-6 \%, p=0.010)$ but not LL-BFR $(-1 \pm 3 \mathrm{~cm}[95 \% \mathrm{CI}:-3$ to -1$],-4 \%$,

$502 p=0.099)$. Jump height recovered to pre-exercise levels in both groups 24 to $48 \mathrm{~h}$ post-

503 exercise (all $p>0.05)$.

504

505 Thigh volume and soreness perceptions. No time* group interaction existed for thigh volume $506(p=0.514)$, however time effects were observed $(p<0.001)$. Thigh volume increased from pre507 to post-exercise after HL-RT (320 ml [95\% CI: 105 to 536], 4.0\%, $p<0.001)$ and LL-BFR 508 (445 ml [95\% CI: 288 to 600], 5.6\%,p<0.001), but returned to pre-exercise levels 2 to $48 \mathrm{~h}$ 509 post-exercise in both groups. A time* group interaction $(p=0.008)$ and time effect $(p<0.001)$

510 for perceived soreness existed. Soreness perceptions increased from pre to post exercise for

511 HL-RT and LL-BFR (both $p<0.001$ ). Perceptions of soreness remained elevated 2 to $48 \mathrm{~h}$

512 after HL-RT (all $p<0.001$ ), and 2 to $24 \mathrm{~h}$ after LL-BFR ( $p<0.001$ to 0.009 ) yet tended to 513 decrease by $48 \mathrm{~h}(p=0.071)$. Soreness perceptions were greater at $24 \mathrm{~h}(p=0.008)$ and $48 \mathrm{~h}$ $514(p=0.022)$ following HL-RT, compared with LL-BFR.

515

\section{DISCUSSION}

This study compared the chronic adaptations and acute responses in skeletal muscle

518 between resistance training with high loads, versus low loads combined with BFR. The key

519 findings were that: (i) in strength-trained individuals, comparable skeletal muscle

520 hypertrophy can be obtained with both LL-BFR, and HL-RT; and (ii) the activation of several

521 signaling proteins involved in mTOR and MAPK pathways, and expression of transcriptional

522 factors involved in initiating ribosome biogenesis, are increased similarly in the short term

523 after LL-BFR and HL-RT, which may account (in part) for the comparable chronic

524 adaptations. Most acute responses and chronic adaptations were similar between the two

525 conditions; however, some important differences were observed. The increase in squat 1RM 
was greater in HL-RT than in LL-BFR, with some evidence suggesting the same trend for maximal knee extension torque.

Chronic hypertrophic responses. Our findings support our primary hypothesis, and reinforce the current consensus that marked skeletal muscle hypertrophy can be achieved with LL-BFR $(9,10,12,28)$. Importantly, the magnitude of hypertrophy was comparable to that observed after resistance training with much higher loads. Although this has been demonstrated previously $(9-11,37)$, these studies have utilized untrained participants. Importantly, and a novelty of the present study, is that this evidence has been attained from a trained cohort, identifying that these individuals can also achieve robust hypertrophy with LL- BFR, akin to HL-RT.

Hitherto, it was unclear whether the acute processes within muscle responsible for these hypertrophic adaptations were similar between HL-RT versus LL-BFR. The only other studies to have investigated this to date, noted that myofibrillar protein synthesis (13) and expression of genes related to muscle function and plasticity (10) were similarly elevated after LL-BFR and HL-RT in untrained individuals. Although we did not directly assess myofibrillar protein synthesis, we found that key protein kinases involved in the mTOR and MAPK pathways (which are involved in myofibrillar protein synthesis $(6,21,22)$ ) responded similarly following LL-BFR and HL-RT exercise. Likewise, we demonstrated that expression of ribosomal RNA transcriptional factors increased following both LL-BFR and HL-RT, which may also enhance muscle protein synthesis rates. Importantly, these cellular and molecular responses to a single session were demonstrated in resistance trained individuals, and in trained skeletal muscle (i.e., following the chronic training period), which likely provides a truer reflection of the nature and magnitude of these anabolic processes than in a naïve state (i.e., before the chronic training period) (10). 
552 Progression in volume-load. It is worth noting that the progression in volume-load across the 553 training period was significantly greater with LL-BFR compared to HL-RT. A previous study 554 involving BFR training observed volume-load to increase moreso with HL-RT compared to 555 the LL-BFR conditions (38). However, in this study, sets were carried out until muscular 556 failure and loads were not progressed during the training block. Instead, volume-load was 557 increased by increasing the number of repetions only, which may explain the divergent 558 findings. In a design similar to the present study, where set and repetition schemes were fixed 559 and the magnitude of loading was progressed, Schoenfeld et al. (39) observed a greater 560 increase in volume-load following LL-RT (without BFR) compared with HL-RT. Thus, it 561 appears that compared to higher load protocols, lower load protocols (with or without BFR)

562 inherently permit greater progression in loads (thereby enhancing volume-load) across a 563 training period.

564 Interestingly, previous literature suggests that RT protocols that comprise of higher 565 volume-loads stimulate greater muscle protein synthesis than lower volume-load protocols 566 (40). The findings from our study do not align with these previous findings, because the acute 567 cellular and molecular responses, and chronic hypertrophic responses were comparable 568 between LL-BFR and HL-RT. In the present study, our HL-RT protocol included multiple 569 exercises, and lower loading conditions (75\% vs 90\% 1RM) than that of Burd et al. (40).

570 Therefore, volume-load with HL-RT was still substantial, despite being less than LL-BFR. It

571 is likely that a threshold for volume-load exists, over which greater volume-loads do not

572 confer greater acute protein synthetic responses, or chronic hypertrophy $(38,39,40)$.

573

574 Acute anabolic signaling responses. Despite the previously reported similar net responses in 575 protein synthesis (13) and chronic hypertrophy $(9-11,37)$ after these training modalities, the 
576 contributions of distinct intracellular pathways (i.e., mTOR vs MAPK) to achieve these net

577 responses have not been examined in this context. Herein, phosphorylation of $\mathrm{p} 70 \mathrm{~S} 6 \mathrm{~K}^{\mathrm{Thr} 389}$

578 appeared to be especially robust following HL-RT (33-fold increase at $2 \mathrm{~h}$ post-exercise),

579 which has been strongly correlated to chronic increases in muscle CSA (27). A higher

580 temporal response in p70S6K phosphorylation tended to occur after HL-RT over the $48 \mathrm{~h}$

581 period $(p=0.064, d=0.89)$, from phosphorylation remaining above rest throughout. This may

582 be expected, as phosphorylation of $\mathrm{p} 70 \mathrm{~S} 6 \mathrm{~K}$ at the $\mathrm{Thr}^{389}$ site is particularly responsive to

583 mechanical stimuli $(22,23,42)$. The different loading conditions imposed between groups in

584 the present study may therefore explain the prolonged phosphorylation of p70S6K, and

585 increase in total p70S6K protein content following HL-RT. Despite this nuance, the chronic

586 changes in muscle CSA were similar between HL-RT and LL-BFR in the present study.

587 Thus, stimuli other than purely mechanical tension may promote skeletal muscle hypertrophy

588 in the long term (5-7), and metabolites themselves may possess anabolic properties instead of

589 purely serving to enhance muscle fiber recruitment (and potentially operate through a

590 mechanism independent of p70S6K). Indeed, both in vivo and in vitro studies have observed

591 the application of lactate (in the absence of exercise) to stimulate ERK1/2 and mTOR

592 signaling pathways acutely (43), and myotube formation with increases in fiber diameter and

593 myonuclei content, respectively (44). Other stimuli that are associated with hypertrophy

594 include hypoxia, metabolite accumulation, and cell swelling (7), all of which are induced by

595 BFR exercise.

596 ERK1/2 phosphorylation significantly increased after LL-BFR, and although this

597 response did not seem to be group-dependent (no interaction), a large effect was observed

598 with LL-BFR $(d=1.22)$ rather than HL-RT $(d=0.25)$. ERK1 and -2 (ERK1/2) are components

599 of the MAPK family, which is known to respond to stress-related external stimuli, and to be

600 particularly responsive to lower load, higher volume protocols than higher load, lower 
601 volume resistance training protocols (45). Recent work in mice suggests a role for ERK1/2 in 602 the regulation of fiber type-specific gene programs. Specifically, ERK1/2 appears to induce a 603 type I, oxidative fiber phenotype in developing mouse muscle (46). Therefore, it may be that

604 stress-related stimuli associated with exercise, such as tissue hypoxia and/or higher metabolic 605 demands $(5,8)$ act as stimuli for the ERK1/2 pathway. For example, injection of exogenous 606 lactate increases ERK1/2 phosphorylation 3.5-fold in mouse muscle (43). Despite these data, 607 the present study did not find a difference between LL-BFR and HL-RT in ERK1/2 608 phosphorylation, and may have been underpowered to detect a such interaction. Future work 609 should seek to expand on ERK1/2 (and other MAPK signalling pathways) responses to LL610 BFR and HL-RT protocols.

611

612 Fiber-type specific responses. In contrast to our hypothesis, we did not observe a preference 613 for type I fiber adaptation with LL-BFR, nor a preference for type II fiber adaptations with 614 HL-RT. Both training groups increased the CSA of type II fibers, whereas only HL-RT 615 increased myonuclear number. Previous studies report that type I fibers are subjected to 616 greater stress during LL-BFR exercise $(18,19)$, and this appears to translate chronically into 617 increased capillarisation and fiber area in type I fibers $(16,17)$. The lack of change in 618 myonuclear accretion with LL-BFR is also interesting, as this contrasts with previous 619 literature $(16-18,28)$. Although robust hypertrophy following LL-BFR has been reported in 620 the absence of myonuclear accretion (10). The lack of type I fiber adaptations following BFR 621 in the present study may relate to the BFR protocol and method of progressive overload that 622 we used. To allow for a more ecologically valid comparison of HL-RT and LL-BFR training 623 protocols, and to eliminate the inherent bias that occurs when attempting to match volume 624 between low-load and high-load conditions (31), we chose a BFR protocol that minimized 625 muscular failure (using three to four repetitions in reserve), and kept volume (sets $\times$ reps) 
626 constant, while progressing the external loading. By the final week of training, some

627 participants were exercising with loads that equated to $\sim 50 \%$ of their baseline $1 \mathrm{RM}$. Thus, the

628 nature of the stimulus likely differed to that of previous studies (16-19), which used lower

629 loading conditions (bodyweight to $20 \% 1 \mathrm{RM}$ ), and/or completed some (or all) sets until

630 muscular failure. Such conditions were likely associated with longer ischemic periods, which

631 severely fatigues type II fibers and relies on the superior recovery rate of type I fibers to

632 maintain force output (14). Despite this, our results indicate a similar hypertrophy of type I

633 and type II fibers with LL-BFR (10\% vs. 13\%), and a more pronounced type II fiber

634 hypertrophy with HL-RT (2\% vs. $18 \%)$. Therefore, it may be advisable to use lower loading

635 conditions $(<20 \% 1 \mathrm{RM})$ and complete some or all sets until muscular failure if the aim is to

636 preferentially target type I fiber adaptations.

637

638 Acute ribosomal RNA responses. These molecular responses represent the initiation of

639 ribosome biogenesis, which is requisite for the increased capacity for protein synthesis that

640 occurs with muscle hypertrophy $(25,26)$. Following resistance exercise, increased

641 transcription of rDNA occurs $(24,47)$, and this is responsible for the accumulation of rRNA,

642 which is believed to precede hypertrophy (25). In the present study, we observed a

643 comparable increase in expression of some of the key, early transcriptional factors including

644 c-Myc, TIF-1A and TAF-1A following exercise, between LL-BFR and HL-RT. Interestingly,

645 neither group demonstrated a significant increase in total RNA or expression of pre-rRNA or

646 rRNA transcripts in the $48 \mathrm{~h}$ following exercise, despite the robust hypertrophy observed with

647 training. This lack of change in rRNA components may be because (i) the participants in this

648 study were trained, and (ii) the participants completed the acute study following 9 weeks of

649 training. In support of this notion, Nader et al. (48) report that ribosomal gene expression is

650 attenuated in trained skeletal muscle. Nonetheless, these gene responses are in alignment with 
651

652

653

654

655

656

657

658

659

660

661

662

663

664

665

666

667

668

669

670

671

672

673

674

675

the previously discussed cellular signaling responses. Collectively, these data suggest that acute molecular responses in muscle were similar between LL-BFR and HL-RT, which may account for the similar muscle hypertrophy between these groups in the long term.

Chronic muscle function responses. From a practical standpoint, the maintenance and increase in strength and power qualities in trained individuals, despite the exclusive performance of BFR, is of importance. Previous studies assessing how BFR affects muscular strength and power often include untrained participants $(9-11,13,37)$. This choice of untrained participants makes it difficult to translate findings to well-trained cohorts, who possess a lower adaptive potential. The few studies that have included athletic or trained cohorts have examined LL-BFR in conjunction with traditional HL-RT (instead of LL-BFR exclusively). These studies found either small increases in strength $(16,49)$, or maintenance of strength and power qualities (50) when LL-BFR was performed, which aligns with the findings of the present study.

Direct comparisons of strength outcomes between LL-BFR and HL-RT is an interesting area. There are consistent reports that HL-RT produces superior strength gains to LL-BFR, particularly when strength is assessed using dynamic tests (e.g. 1RM) $(12,16,38)$. Even when seemingly non-specific tests (e.g. isometric MVC) are used, HL-RT may still be more effective at eliciting strength gains (12). The findings of the present study appear to support this, because isometric strength did not increase with LL-BFR, and squat 1RM increased more with HL-RT. However, some researchers have postulated that such strength differences may be influenced by the strength tests themselves, which inherently favor HLRT as external loads used in training are much greater than LL-BFR $(9,51)$. Indeed, there is evidence to suggest that more opportunities to perform strength assessments (i.e., practice) may attenuate these differences in strength (51). With that said, in the present study subjects 
676 performed the strength assessments on four occasions (familiarization, baseline, mid-testing, 677 post-testing), and notable differences in strength were still observed. Although it is likely that 678 test specificity did contribute somewhat to these findings, we believe there is a stronger 679 physiological contribution to the divergent strength adaptations observed in the present study, 680 and many previous studies $(12,16,38)$. For example, adaptations within the nervous system 681 that influence central drive, including motor unit recruitment, firing rates, and 682 synchronization, may have been inferior with LL-BFR due to the lower loading conditions used (14). In particular, the inhibitory influence of group III/IV afferents — which are thought 684 to be strongly activated during ischemic exercise $(8,14,16)$-might limit the firing rates of 685 the highest threshold motor units, thereby limiting maximal strength performance (14). This neural adaptation theory may also reveal why studies including more strength 687 assessments report similar increases in strength between LL-BFR and HL-RT. Frequent 688 maximal strength assessments would stimulate maximal motor unit recruitment, firing rates and/or synchronization, and offset potential differences between LL-BFR and HL-RT protocols, when compared with isolated pre- and post-training assessments. Neural

691 adaptations were not assessed in the present study, so we can only speculate if this 692 contributed to the inferior strength adaptations with LL-BFR. Another suggested explanation 693 for the observed differences in strength and power qualities may be related to mechanical or 694 morphological properties of tendons (37). Although such differences were questioned in a 695 recent study (9), we cannot exclude differences in mechanical properties in both muscle and 696 tendons as possible causes for the distinct functional adaptations in this study. Future research 697 should seek to investigate motor unit recruitment and voluntary activation adaptations as well 698 as differences in tissue mechanical properties following chronic periods of LL-BFR and HL699 RT. 
701 Acute muscle function \& recovery. Muscular performance was also evaluated following the 702 acute session to gain insight into the fatigue and recovery timelines for each mode of 703 exercise. We report similar significant impairments in both isometric strength and 704 countermovement jump performance after LL-BFR and HL-RT, immediately following 705 exercise, and at $2 \mathrm{~h}$ post-exercise. Performance in both assessments recovered to baseline

706 levels at $24 \mathrm{~h}$. While there are some accounts of prolonged impairments in muscle function 707 extending past $24 \mathrm{~h}$ with both LL-BFR and HL-RT $(52,53)$, much of the previous literature suggest these decrements in performance are due to fatigue, given they subside in the hours following exercise (54-57). Discrepancies between outcomes are likely due to the exercise load and volume used, with higher volumes (e.g. 5 sets) or sets performed to muscular

711 failure, resulting in prolonged strength impairments $(52,53)$. In the present study, although 712 volume was relatively high (3 exercises, 3-4 sets), we used a submaximal repetition scheme

713 (30-15-15-15). Moreover, we aimed to standardize proximity to failure using the RIR method. It is also pertinent to note in the present study that these assessments were completed after 9 weeks of exposure to an identical (albeit progressive) stimulus to that used in the preceding training period. Therefore, participants were well accustomed to the respective

717 stimuli of high- or low-loads with BFR, unlike the cited example, which involved participants

718 new to strength training $(52,53)$. We solely quantified the peak torque or jump height

719 produced by the individual, which is a limitation of the present study. Ultimately, this

720 provides an integrated measure of fatigue, but cannot distinguish between peripheral or central origins of such fatigue (58). Previous studies report severe peripheral fatigue with

722 BFR which promptly recovers $(<8 \mathrm{~min})$ upon reperfusion to match the degree of impairment 723 that occurs with HL-RT (55). Alongside measures of muscle function, we also inferred muscle damage indirectly 725 through thigh swelling and perceptions of soreness. Soreness ratings were greater at 24, and 
$72648 \mathrm{~h}$ following HL-RT, which contrasts the findings of Brander et al. (59), who reported

727 higher perceived soreness following both continuous and intermittent LL-BFR protocols

728 compared with HL-RT. The discrepancy is likely due to the use of untrained participants in

729 that study, whereas the 9-week training period in the present study presumably induced a

730 repeated bout effect (52). Thigh swelling measurements were comparable between exercise

731 protocols throughout the $48 \mathrm{~h}$ recovery period, which corroborates previous findings (52).

732 Collectively, the muscle function, circumference and soreness data suggest that there were

733 similar fatigue and recovery timelines between LL-BFR and HL-RT, which aligns with

734 previous literature $(52,54)$. While muscular function and swelling may recover to baseline

735 within $24 \mathrm{~h}$ following LL-BFR exercise, it is not clear if other processes within skeletal

736 muscle are also restored within this time frame to support optimal performance in subsequent

737 exercise sessions. Therefore, the recovery period between LL-BFR training sessions is an

738 important consideration. Although a period of $24 \mathrm{~h}$ appears to be sufficient, further research

739 on this topic is necessary before definitive conclusions can be drawn.

740

741 Considerations. A 'traditional' non-exercise control group was not included in the present

742 study. Without this, it cannot be confirmed that the outcome measures displaying a time

743 effect increased because of the training program per se. However, all variables included are

744 known to increase with resistance training, and the magnitude of effects are commensurate

745 with those previously reported $(9,10,12,13)$. The aim of the present study was not to assess

746 the efficacy of LL-BFR. Instead, it was to compare LL-BFR to protocols with greater

747 ecological validity, hence why the HL-RT group was included over a non-exercise or low-

748 load non-BFR control.

749 Both male and female participants were recruited in the present study. We view this

750 as both a strength and a limitation. Young female participants are underrepresented in BFR 
751 literature (60), which makes it difficult to speculate if responses to BFR exercise may differ

752 between sexes. In the present study, analyses of sex-specific responses were not conducted as

753 this fall outside the scope of the investigation. Moreover, the inclusion of invasive

754 measurement techniques, such as muscle biopsies, limited our sample size; thus, the study is

755 insufficiently powered to evaluate training responses between sexes. Nevertheless, although

756 training responses between males and females may differ in absolute terms, it appears these

757 are similar when expressed relatively (61). For this reason, many outcome measures in the

758 present study are expressed as a percentage change from baseline. Finally, we acknowledge it

759 is possible that the menstrual cycle may exert some influence on training adaptations (60). To

760 mitigate any potential impact, we stratified female participants into training groups based on

761 contraceptive use, and method of contraceptive (e.g. hormonal, intrauterine etc.) to ensure an

762 equal distribution across groups.

763

764 In conclusion, we compared the chronic and acute effects of LL-BFR and HL-RT, to

765 evaluate the influence of resistance exercise protocols differing in contributions of

766 mechanical versus metabolic stimuli on skeletal muscle characteristics. The findings

767 supported our primary hypothesis, that the greater metabolic stimulus provided by LL-BFR

768 can compensate for reduced mechanical loading and induce significant skeletal muscle

769 hypertrophy, commensurate with that observed after HL-RT. The acute signaling and

770 transcriptional responses within muscle that are responsible for such changes in hypertrophy

771 also appear similar, yet particular signaling pathways may be more active than others,

772 depending on the nature of the dominant external stimulus e.g., mechanical stretch versus

773 tissue hypoxia, and should be further explored in future investigations. Examining these

774 training protocols in isolation has permitted an understanding of which functional properties

775 may develop/diminish after either training mode. Higher loading conditions appear necessary 
to maximize muscular strength adaptations. Despite this, lower loads combined with BFR permit the maintenance of muscular strength and power in trained individuals. Ultimately, these findings encourage future studies to combine these protocols to distinguish whether they pose a synergistic effect in skeletal muscle.

\section{ENDNOTE}

At the request of the author(s), readers are herein alerted to the fact that additional materials related to this manuscript may be found at https://doi.org/10.6084/m9.figshare.15167739.v1. These materials are not a part of this manuscript and have not undergone peer review by the American Physiological Society (APS). APS and the journal editors take no responsibility for these materials, for the website address, or for any links to or from it.

\section{ACKNOWLEDGEMENTS}

This research was supported by an Australian Government Research Training Program (RTP) Scholarship to CD. The study was further supported by the Queensland Academy of Sport's Sport Performance Innovation and Knowledge Excellence (SPIKE) unit, the Swedish Research Council for Sport Science and the Swedish Military Research Authority (\#AF 922 0916). The authors declare they have no conflicts of interest.

\section{AUTHOR CONTRIBUTIONS}

The studies within this manuscript were performed at Department of Physical Performance at the Norwegian School of Sport Science, in Oslo, Norway. CD was involved in the conception and design of the work, acquisition, analysis, and interpretation of the data, and in the preparation and drafting of the manuscript. TCN, MM, KTC, OH, NP, and BE were involved in the acquisition, analysis and interpretation of the data, and in the preparation and drafting 
801 of the manuscript. JSC, JP TR, and LAR were involved in the conception and design of the 802 work, interpretation of the data, and in the drafting of the manuscript. All authors approved 803 the final version of the manuscript and all agree to be accountable for all aspects of the work

804 in ensuring that questions related to the accuracy or integrity of any part of the work are

805 appropriately investigated and resolved. All persons designated as authors qualify for

806 authorship, and all those who qualify for authorship are listed.

807

808

REFERENCES

809 1. ACSM. American College of Sports Medicine position stand. Progression models in 810 resistance training for healthy adults. Med Sci Sports Exerc 41: 687-708, 2009.

811 doi:10.1249/MSS.0b013e3181915670

812 2. Schoenfeld BJ. Science and development of muscle hypertrophy. Champaign, IL:

813 Human Kinetics, 2016.

814 3. Williams TD, Tolusso DV, Fedewa MV, and Esco MR. Comparison of periodized

815 and non-periodized resistance training on maximal strength: A meta-analysis. Sports Med 47:

816 2083-2100, 2017. doi:10.1007/s40279-017-0734-y

817 4. Hughes L, Paton B, Rosenblatt B, Gissane C, and Patterson SD. Blood flow 818 restriction training in clinical musculoskeletal rehabilitation: a systematic review and meta819 analysis. Br J Sports Med 2017. doi:10.1136/bjsports-2016-097071

820 5. Takada S, Okita K, Suga T, Omokawa M, Kadoguchi T, Sato T, Takahashi M, 821 Yokota T, Hirabayashi K, Morita N, Horiuchi M, Kinugawa S, and Tsutsui H. Low-

822 intensity exercise can increase muscle mass and strength proportionally to enhanced

823 metabolic stress under ischemic conditions. J Appl Physiol (1985) 113: 199-205, 2012.

824 doi:10.1152/japplphysiol.00149.2012

825 6. Wackerhage HS, B J.; Hamilton, D L.; Lehti, M.; Hulmi, J J. . Stimuli and 826 sensors that initiate skeletal muscle hypertrophy following resistance exercise. $J$ Appl Physiol 827 126: 30-43, 2019. doi:10.1152/japplphysiol.00685.2018

828 7. Pearson SJ, and Hussain SR. A review on the mechanisms of blood-flow restriction 829 resistance training-induced muscle hypertrophy. Sports Med 45: 187-200, 2015.

830 doi:10.1007/s40279-014-0264-9 
831 8. Suga T, Okita K, Takada S, Omokawa M, Kadoguchi T, Yokota T, Hirabayashi

832 K, Takahashi M, Morita N, Horiuchi M, Kinugawa S, and Tsutsui H. Effect of multiple

833 set on intramuscular metabolic stress during low-intensity resistance exercise with blood flow

834 restriction. Eur J Appl Physiol 112: 3915-3920, 2012. doi:10.1007/s00421-012-2377-x

835 9. Centner C, Lauber B, Seynnes OR, Jerger S, Sohnius T, Gollhofer A, and König

836 D. Low-load blood flow restriction training induces similar morphological and mechanical

837 Achilles tendon adaptations compared with high-load resistance training. J Appl Physiol 127:

838 1660-1667, 2019. doi:10.1152/japplphysiol.00602.2019

839 10. Ellefsen S, Hammarstrom D, Strand TA, Zacharoff E, Whist JE, Rauk I,

840 Nygaard H, Vegge G, Hanestadhaugen M, Wernbom M, Cumming KT, Ronning R,

841 Raastad T, and Ronnestad BR. Blood flow-restricted strength training displays high

842 functional and biological efficacy in women: a within-subject comparison with high-load

843 strength training. Am J Physiol Reg Integr Comp Physiol 309: R767-779, 2015.

844 doi:10.1152/ajpregu.00497.2014

845 11. Kubo K, Komuro T, Ishiguro N, Tsunoda N, Sato Y, Ishii N, Kanehisa H, and

846 Fukunaga T. Effects of low-load resistance training with vascular occlusion on the

847 mechanical properties of muscle and tendon. J Appl Biomech 22: 112-119, 2006.

848 doi:doi:10.1123/jab.22.2.112

849 12. Lixandrao ME, Ugrinowitsch C, Berton R, Vechin FC, Conceicao MS, Damas F, 850 Libardi CA, and Roschel H. Magnitude of muscle strength and mass adaptations between

851 high-load resistance training versus low-load resistance training associated with blood-flow

852 restriction: A systematic review and meta-analysis. Sports Med 2017. doi:10.1007/s40279-

853 017-0795-y

854 13. Sieljacks P, Wang J, Groennebaek T, Rindom E, Jakobsgaard JE, Herskind J,

855 Gravholt A, Moller AB, Musci RV, de Paoli FV, Hamilton KL, Miller BF, and Vissing

856 K. Six weeks of low-load blood flow restricted and high-load resistance exercise training

857 produce similar increases in cumulative myofibrillar protein synthesis and ribosomal

858 biogenesis in healthy males. Front Physiol 10: 649, 2019. doi:10.3389/fphys.2019.00649

859 14. Wernbom M, and Aagaard P. Muscle fibre activation and fatigue with low-load

860 blood flow restricted resistance exercise - An integrative physiology review. Acta Physiol

861 (Oxf) e13302, 2019. doi:10.1111/apha.13302

862 15. Karabulut M, Abe T, Sato Y, and Bemben MG. The effects of low-intensity

863 resistance training with vascular restriction on leg muscle strength in older men. Eur J Appl

864 Physiol 108: 147-155, 2010. doi:10.1007/s00421-009-1204-5 
865 16. Bjornsen T, Wernbom M, Kirketeig A, Paulsen G, Samnoy L, Baekken L,

866 Cameron-Smith D, Berntsen S, and Raastad T. Type 1 muscle fiber hypertrophy after

867 blood flow-restricted training in powerlifters. Med Sci Sports Exerc 51: 288-298, 2019.

868 doi:10.1249/mss.0000000000001775

869 17. Jakobsgaard JE, Christiansen M, Sieljacks P, Wang J, Groennebaek T, de Paoli

870 F, and Vissing K. Impact of blood flow-restricted bodyweight exercise on skeletal muscle

871 adaptations. Clin Physiol Funct Imaging 2018. doi:10.1111/cpf.12509

872 18. Bjornsen T, Wernbom M, Lovstad AT, Paulsen G, D'Souza RF, Cameron-Smith

873 D, Flesche A, Hisdal J, Berntsen S, and Raastad T. Delayed myonuclear addition,

874 myofiber hypertrophy and increases in strength with high-frequency low-load blood flow

875 restricted training to volitional failure. J Appl Physiol (1985) 2018.

876 doi:10.1152/japplphysiol.00397.2018

877 19. Cumming KT, Paulsen G, Wernbom M, Ugelstad I, and Raastad T. Acute

878 response and subcellular movement of HSP27, alphaB-crystallin and HSP70 in human

879 skeletal muscle after blood-flow-restricted low-load resistance exercise. Acta Physiol (Oxf)

880 211: 634-646, 2014. doi:10.1111/apha.12305

881 20. Häkkinen K, Alén M, and Komi PV. Neuromuscular, anaerobic, and aerobic

882 performance characteristics of elite power athletes. Eur J Appl Physiol Occup Physiol 53: 97-

883 105, 1984. doi:10.1007/BF00422570

884 21. Chau Long Y, Widegren U, and Zierath JR. Exercise-induced mitogen-activated

885 protein kinase signalling in skeletal muscle. Proc Nutr Soc 63: 227-232, 2004.

886 doi:10.1079/PNS2004346

887 22. Hornberger TA, Sukhija KB, Wang XR, and Chien S. mTOR is the rapamycin-

888 sensitive kinase that confers mechanically-induced phosphorylation of the hydrophobic motif

889 site $\operatorname{Thr}(389)$ in p70(S6k). FEBS letters 581: 4562-4566, 2007.

890 doi:10.1016/j.febslet.2007.08.045

891 23. Spangenburg EE, and McBride TA. Inhibition of stretch-activated channels during

892 eccentric muscle contraction attenuates p70S6K activation. J Appl Physiol (1985) 100: 129-

893 135, 2006. doi:10.1152/japplphysiol.00619.2005

894 24. Figueiredo VC, Caldow MK, Massie V, Markworth JF, Cameron-Smith D, and

895 Blazevich AJ. Ribosome biogenesis adaptation in resistance training-induced human skeletal

896 muscle hypertrophy. Am J Physiol Endocrinol Metab 309: E72-83, 2015.

897 doi:10.1152/ajpendo.00050.2015 
898

899

900

901

902

903

904

905

906

907

908

909

910

911

912

913

914

915

916

917

918

919

920

921

922

923

924

925

926

927

928

929

930

25. Kim H-G, Guo B, and Nader GA. Regulation of ribosome biogenesis during skeletal muscle hypertrophy. Exerc Sport Sci Rev 47: 91-97, 2019. doi:10.1249/jes.0000000000000179

26. Nakada S, Ogasawara R, Kawada S, Maekawa T, and Ishii N. Correlation between ribosome biogenesis and the magnitude of hypertrophy in overloaded skeletal muscle. PloS one 11: e0147284, 2016. doi:10.1371/journal.pone.0147284

27. Baar K, and Esser K. Phosphorylation of p70(S6k) correlates with increased skeletal muscle mass following resistance exercise. Am J Physiol 276: C120-127, 1999. doi:10.1152/ajpcell.1999.276.1.C120

28. Nielsen JL, Aagaard P, Bech RD, Nygaard T, Hvid LG, Wernbom M, Suetta C, and Frandsen U. Proliferation of myogenic stem cells in human skeletal muscle in response to low-load resistance training with blood flow restriction. J Physiol 590: 4351-4361, 2012. doi:10.1113/jphysiol.2012.237008

\section{Roberts LA, Raastad T, Markworth JF, Figueiredo VC, Egner IM, Shield A,}

Cameron-Smith D, Coombes JS, and Peake JM. Post-exercise cold water immersion attenuates acute anabolic signalling and long-term adaptations in muscle to strength training. J Physiol 593: 4285-4301, 2015. doi:10.1113/jp270570

30. Norton K CJ, Parker R, Williams A, Hobson-Powell A, Knox C. . New Australian standard for adult pre-exercise screening. Sport Health 12-18, 2012.

31. Schoenfeld BJ, Grgic J, Ogborn D, and Krieger JW. Strength and hypertrophy adaptations between low- versus high-load resistance training: A systematic review and metaanalysis. J Strength Cond Res 2017. doi:10.1519/jsc.0000000000002200

32. Katch VL, and Katch FI. A simple anthropometric method for calculating segmental leg limb volume. Res Q Am Assoc Health Phys Ed Recr 45: 211-214, 1974. doi:10.1080/10671188.1974.10615262

33. Benjamini Y, and Hochberg Y. Controlling the false discovery rate: a practical and powerful approach to multiple testing. J R Stat Soc Series B Methodol 57: 289-300, 1995.

34. Glickman ME, Rao SR, and Schultz MR. False discovery rate control is a recommended alternative to Bonferroni-type adjustments in health studies. J Clin Epidemiol 67: 850-857, 2014. doi:10.1016/j.jclinepi.2014.03.012

35. Dankel SJ, and Loenneke JP. Effect sizes for paired data should use the change score variability rather than the pre-test variability. J Strength Cond Res 35: 1773-1778, 2021. doi:10.1519/jsc.0000000000002946 
931 36. Cohen J. Statistical power analysis for the behavioral sciences. 2nd. Hillsdale, NJ:

932 erlbaum, 1988.

933 37. Madarame H, Ochi E, Tomioka Y, Nakazato K, and Ishii N. Blood flow-restricted

934 training does not improve jump performance in untrained young men. Acta Physiol Hung 98:

935 465-471, 2011. doi:10.1556/APhysiol.98.2011.4.10

936 38. Jessee MB, Buckner SL, Mouser JG, Mattocks KT, Dankel SJ, Abe T, Bell ZW,

937 Bentley JP, and Loenneke JP. Muscle adaptations to high-load training and very low-load

938 training with and without blood flow restriction. Frontiers Physiol 9: 1448, 2018.

939 doi:10.3389/fphys.2018.01448

940 39. Schoenfeld BJ, Ogborn D, Contreras B, Cappaert T, Silva Ribeiro A, Alvar BA, 941 and Vigotsky AD. A comparison of increases in volume load over 8 weeks of low-versus

942 high-load resistance training. Asian J Sports Med 7: e29247, 2016. doi:10.5812/asjsm.29247

943 40. Burd NA, West DW, Staples AW, Atherton PJ, Baker JM, Moore DR, Holwerda

944 AM, Parise G, Rennie MJ, Baker SK, and Phillips SM. Low-load high volume resistance

945 exercise stimulates muscle protein synthesis more than high-load low volume resistance

946 exercise in young men. PloS one 5: e12033, 2010. doi:10.1371/journal.pone.0012033

947 41. Loenneke JP, Fahs CA, Wilson JM, and Bemben MG. Blood flow restriction: the

948 metabolite/volume threshold theory. Med Hypotheses 77: 748-752, 2011.

949 doi:10.1016/j.mehy.2011.07.029

950 42. Burry M, Hawkins D, and Spangenburg EE. Lengthening contractions

951 differentially affect p70s6k phosphorylation compared to isometric contractions in rat skeletal

952 muscle. Eur J Appl Physiol 100: 409-415, 2007. doi:10.1007/s00421-007-0444-5

953 43. Cerda-Kohler H, Henríquez-Olguín C, Casas M, Jensen TE, Llanos P, and

954 Jaimovich E. Lactate administration activates the ERK1/2, mTORC1, and AMPK pathways

955 differentially according to skeletal muscle type in mouse. Physiol Rep 6: e13800, 2018.

956 doi:10.14814/phy2.13800

957 44. Ohno Y, Ando K, Ito T, Suda Y, Matsui Y, Oyama A, Kaneko H, Yokoyama S,

958 Egawa T, and Goto K. Lactate stimulates a potential for hypertrophy and regeneration of

959 mouse skeletal muscle. Nutrients 11: 869, 2019. doi:10.3390/nu11040869

960 45. Hulmi JJ, Walker S, Ahtiainen JP, Nyman K, Kraemer WJ, and Hakkinen K.

961 Molecular signaling in muscle is affected by the specificity of resistance exercise protocol.

962 Scand J Med Sci Sports 22: 240-248, 2012. doi:10.1111/j.1600-0838.2010.01198.x

963 46. Boyer JG, Prasad V, Song T, Lee D, Fu X, Grimes KM, Sargent MA,

964 Sadayappan S, and Molkentin JD. ERK1/2 signaling induces skeletal muscle slow fiber- 
965 type switching and reduces muscular dystrophy disease severity. JCI Insight 5: 2019.

966 doi:10.1172/jci.insight.127356

967 47. Figueiredo VC, Roberts LA, Markworth JF, Barnett MP, Coombes JS, Raastad

968 T, Peake JM, and Cameron-Smith D. Impact of resistance exercise on ribosome biogenesis

969 is acutely regulated by post-exercise recovery strategies. Physiol Rep 4: 2016.

970 doi:10.14814/phy2.12670

971 48. Nader GA, von Walden F, Liu C, Lindvall J, Gutmann L, Pistilli EE, and

972 Gordon PM. Resistance exercise training modulates acute gene expression during human

973 skeletal muscle hypertrophy. J Appl Physiol (1985) 116: 693-702, 2014.

974 doi:10.1152/japplphysiol.01366.2013

975 49. Luebbers PE, Fry AC, Kriley LM, and Butler MS. The effects of a 7-week

976 practical blood flow restriction program on well-trained collegiate athletes. J Strength Cond

977 Res 28: 2270-2280, 2014. doi:10.1519/jsc.0000000000000385

978 50. Scott BR, Peiffer JJ, and Goods PSR. The effects of supplementary low-load blood

979 flow restriction training on morphological and performance-based adaptations in team sport

980 athletes. J Strength Cond Res 31: 2147-2154, 2017. doi:10.1519/jsc.0000000000001671

981 51. Spitz RW, Bell ZW, Wong V, Yamada Y, Song JS, Buckner SL, Abe T, and

982 Loenneke JP. Strength testing or strength training: considerations for future research.

983 Physiol Meas 41: 09tr01, 2020. doi:10.1088/1361-6579/abb1fa

984 52. Sieljacks P, Matzon A, Wernbom M, Ringgaard S, Vissing K, and Overgaard K.

985 Muscle damage and repeated bout effect following blood flow restricted exercise. Eur J Appl

986 Physiol 116: 513-525, 2016. doi:10.1007/s00421-015-3304-8

987 53. Wernbom M, Paulsen G, Nilsen TS, Hisdal J, and Raastad T. Contractile function

988 and sarcolemmal permeability after acute low-load resistance exercise with blood flow

989 restriction. Eur J Appl Physiol 112: 2051-2063, 2012. doi:10.1007/s00421-011-2172-0

990 54. Hill EC, Housh TJ, Smith CM, Keller JL, Schmidt RJ, and Johnson GO.

991 Eccentric and concentric blood flow restriction resistance training on indices of delayed onset

992 muscle soreness in untrained women. Eur J Appl Physiol 119: 2363-2373, 2019.

993 doi:10.1007/s00421-019-04220-8

994 55. Husmann F, Mittlmeier T, Bruhn S, Zschorlich V, and Behrens M. Impact of

995 blood flow restriction exercise on muscle fatigue development and recovery. Med Sci Sports

996 Exerc 2017. doi:10.1249/mss.0000000000001475 
56. Loenneke JP, Thiebaud RS, Fahs CA, Rossow LM, Abe T, and Bemben MG.

998 Blood flow restriction does not result in prolonged decrements in torque. Eur J Appl Physiol

999 113: 923-931, 2013. doi:10.1007/s00421-012-2502-x

1000 57. Thiebaud RS, Loenneke JP, Fahs CA, Kim D, Ye X, Abe T, Nosaka K, and

1001 Bemben MG. Muscle damage after low-intensity eccentric contractions with blood flow

1002 restriction. Acta Physiol Hung 101: 150-157, 2014. doi:10.1556/APhysiol.101.2014.2.3

1003 58. Bigland-Ritchie B, and Woods JJ. Changes in muscle contractile properties and

1004 neural control during human muscular fatigue. Muscle Nerve 7: 691-699, 1984.

1005 doi:10.1002/mus.880070902

1006 59. Brandner CR, and Warmington SA. Delayed onset muscle soreness and perceived

1007 exertion following blood flow restriction exercise. J Strength Cond Res 2017.

1008 doi:10.1519/jsc.0000000000001779

1009 60. Counts BR, Rossow LM, Mattocks KT, Mouser JG, Jessee MB, Buckner SL,

1010 Dankel SJ, and Loenneke JP. Let's talk about sex: where are the young females in blood

1011 flow restriction research? Clin Physiol Funct Imaging 38: 1-3, 2018. doi:10.1111/cpf.12394

1012 61. Roberts BM, Nuckols G, and Krieger JW. Sex differences in resistance training: A

1013 systematic review and meta-analysis. J Strength Cond Res 34: 2020.

1014

1015 


\section{FIGURE LEGENDS}

1017 Figure 1. Absolute changes in total quadricep cross-sectional area (CSA) from MRI (A), and lower body 1018 lean mass from DXA (B). Solid bars indicate mean pre- vs post-training response in the LL-BFR (black) 1019 and HL-RT (white) group. Error bars indicate standard deviation of group responses. Horizontal lines indicate individual participant responses. $* * *$ indicates a significant change from baseline $(p<0.001)$.

1021

1022

Figure 2. Changes in type I (A) and type II (B) fiber areas and myonuclear number per type I (C) and type II

1023 (D) fibers. Representative immunofluorescence images of muscle fiber membranes stained red for dystrophin (E), and myonuclei stained green for PCM1 (F) and blue for DAPI (G). Nuclei within skeletal muscle were classified as myonuclei if they were positive for PCM1 and DAPI, and the geometrical center was located within the dystrophin ring $(\mathrm{H})$. Scale bar $=100 \mu \mathrm{m}$. Error bars represent standard deviation. $*$ indicates a significant change from baseline $(\mathrm{p}<0.05)$.

1028

1029

Figure 3. Fold changes in phosphorylation of (A) p70S6K at $\mathrm{Thr}^{389}$, (B) rpS6 at $\mathrm{Ser}^{235 / 236}$, (C) 4E-BP1 at $\mathrm{Thr}^{37 / 46}$, and (D) ERK1/2 at $\mathrm{Thr}^{202} / \mathrm{Tyr}^{204}$. (E) Representative immunoblots are provided for each target protein with molecular mass information. ${ }^{* * *}$ indicates a significant change from baseline $(\mathrm{p}<0.001) . * *$ indicates a significant change from baseline $(\mathrm{p}<0.01)$. *indicates a significant change from baseline $(\mathrm{p}<0.05)$. $\dagger$ indicates a significant difference from LL-BFR $(\mathrm{p}<0.05)$. Error bars represent standard deviation

1034

1035

Figure 4. Fold changes in expression of (A) POLR1A mRNA, (B) c-Myc mRNA, (C) TIF-1A mRNA, (D) TAF-1A mRNA, (E) UBF mRNA and (F), 45S pre-rRNA relative to the to the geometric mean of the expression of two housekeeping genes (GAPDH and HPRT). Error bars represent standard deviation. \#indicates significantly different from HL-RT (main effect of group, $\mathrm{p}<0.05$ ). *indicates a significant change from baseline $(\mathrm{p}<0.05)$

1040

Figure 5. Percentage change of (A) total muscle RNA content ( $\mu$ g RNA/mg tissue wet weight) and expression of rRNA transcripts (B) $5.8 \mathrm{~S}$ rRNA, (C) 18S rRNA, (D) 28S rRNA and (E) 45S pre-rRNA relative to the geometric mean of the expression of two housekeeping genes (GAPDH and HPRT). Error bars represent standard deviation

1045

Figure 6. Acute decrements and recovery in (A) knee extension peak torque and (B) countermovement jump height. Data are presented as percent change (\%) from pre-exercise values. Perceived muscle soreness (C) across acute study is presented using CR-10 scale. Error bars indicate standard deviation. ***indicates a significant change from pre-exercise $(p<0.001) .{ }^{*}$ indicates a significant change from pre-exercise $(p<0.01)$.

1051 $*$ indicates a significant change from pre-exercise $(p<0.05)$. †ंindicates a significant difference from LL-BFR 
Table 1. Participant Characteristics

\begin{tabular}{lcc}
\cline { 2 - 3 } & HL-RT & LL-BFR \\
\hline \multicolumn{1}{c}{ Training Study } & $\mathrm{n}=10$ & $\mathrm{n}=11$ \\
Participants & $24.3 \pm 2.9$ & $23.7 \pm 3.1$ \\
Age (years) & $77.4 \pm 12.9$ & $75.5 \pm 10.3$ \\
Body Mass (kg) & $20.3 \pm 3.5$ & $19.4 \pm 3.9$ \\
Lower Body Lean Mass (kg) & $1.4 \pm 0.4$ & $1.3 \pm 0.3$ \\
Relative Squat Strength & & \\
(1RM/BM) & $4.8 \pm 2.8$ & $5.2 \pm 2.9$ \\
Training History (years) & & $\mathrm{n}=8$ \\
\hline \multicolumn{1}{c}{ Acute Study } & $23.8 \pm 2.7$ & $23.9 \pm 3.2$ \\
\hline Participants & $78.1 \pm 14.5$ & $75.9 \pm 10.7$ \\
Age (years) & $20.3 \pm 3.9$ & $19.4 \pm 4.1$ \\
Body Mass (kg) & & $5.3 \pm 0.4$ \\
Lower Body Lean Mass (kg) & & \\
Relative Squat Strength & & \\
(1RM/BM) & & \\
Training History (years) & & \\
\hline & & \\
\hline
\end{tabular}

Data are presented as mean \pm SD. Abbreviations: Training history (years of continuous previous strength training); HL-RT, high-load resistance training; LL-BFR, low-load blood flow restriction; 1RM, one-repetition maximum. 
Table 2. Training Program Overview

Low-Load Blood Flow Restriction (LL-BFR)

High-Load (HL-RT)

\begin{tabular}{|c|c|c|c|c|}
\hline & Repetitions (in set order) & Intensity & Repetitions (in set order) & Intensity \\
\hline \multicolumn{5}{|l|}{ Session One and Three } \\
\hline 1. Squat & $\begin{array}{c}\text { Weeks } 1-4(30,15,15,15 \text { reps }) \\
\text { Week } 5(30,15,15 \text { reps }) \\
\text { Weeks } 6-9(30,15,15,15 \text { reps })\end{array}$ & $\begin{array}{c}\sim 30 \% 1 \mathrm{RM} \text { (adjusted to } \\
\text { maintain } 1-4 \mathrm{RIR})\end{array}$ & $\begin{array}{c}\text { Weeks } 1-4(8,8,8,8 \text { reps }) \\
\text { Week } 5(8,8,8 \text { reps }) \\
\text { Weeks } 6-9(8,8,8,8 \text { reps })\end{array}$ & $\begin{array}{l}\sim 75 \% 1 \mathrm{RM} \text { (adjusted to } \\
\text { maintain } 1-2 \mathrm{RIR} \text { ) }\end{array}$ \\
\hline 2. Leg Press & $\begin{array}{c}\text { Weeks } 1-4(30,15,15,15 \text { reps }) \\
\text { Week } 5(30,15,15 \text { reps }) \\
\text { Weeks } 6-9(30,15,15,15 \text { reps })\end{array}$ & $\begin{array}{c}\sim 30 \% 1 \mathrm{RM} \text { (adjusted to } \\
\text { maintain } 1-4 \mathrm{RIR} \text { ) }\end{array}$ & $\begin{array}{c}\text { Weeks } 1-4(8,8,8,8 \text { reps }) \\
\text { Week } 5(8,8,8 \text { reps }) \\
\text { Weeks } 6-9(8,8,8,8 \text { reps }\end{array}$ & $\begin{array}{l}\sim 75 \% 1 \mathrm{RM} \text { (adjusted to } \\
\text { maintain } 1-2 \mathrm{RIR})\end{array}$ \\
\hline 3. Leg Extension & $\begin{array}{c}\text { Weeks } 1-4(30,15,15,15 \text { reps }) \\
\text { Week } 5(30,15,15 \text { reps }) \\
\text { Weeks } 6-9(30,15,15,15 \text { reps })\end{array}$ & $\begin{array}{c}\sim 30 \% 1 \mathrm{RM} \text { (adjusted to } \\
\text { maintain } 1-4 \mathrm{RIR} \text { ) }\end{array}$ & $\begin{array}{c}\text { Weeks } 1-4(8,8,8,8 \text { reps }) \\
\text { Week } 5(8,8,8 \text { reps }) \\
\text { Weeks } 6-9(8,8,8,8 \text { reps }\end{array}$ & $\begin{array}{l}\sim 75 \% 1 \mathrm{RM} \text { (adjusted to } \\
\text { maintain } 1-2 \mathrm{RIR} \text { ) }\end{array}$ \\
\hline \multicolumn{5}{|l|}{ Session Two } \\
\hline $\begin{array}{l}\text { 1. Bulgarian Split } \\
\text { Squat }\end{array}$ & $\begin{array}{c}\text { Weeks } 1-4(15,15,15,15 \text { reps }) \\
\text { Week } 5(15,15,15 \text { reps }) \\
\text { Weeks } 6-9(15,15,15,15 \text { reps })\end{array}$ & $\begin{array}{l}\sim 15 \% \text { of PTBM (adjusted to } \\
\text { maintain } 1-4 \text { RIR) }\end{array}$ & $\begin{array}{c}\text { Weeks } 1-4(8,8,8,8 \text { reps }) \\
\text { Week } 5(8,8,8 \text { reps }) \\
\text { Weeks } 6-9(8,8,8,8 \text { reps }\end{array}$ & $\begin{array}{c}\sim 30 \% \text { of PTBM (adjusted to } \\
\text { maintain } 1-2 \text { RIR) }\end{array}$ \\
\hline 2. Leg Extension & $\begin{array}{c}\text { Weeks } 1-4(30,15,15,15 \text { reps }) \\
\text { Week } 5(30,15,15 \text { reps }) \\
\text { Weeks } 6-9(30,15,15,15 \text { reps })\end{array}$ & $\begin{array}{c}\sim 30 \% 1 \mathrm{RM} \text { (adjusted to } \\
\text { maintain } 1-4 \mathrm{RIR})\end{array}$ & $\begin{array}{c}\text { Weeks } 1-4(8,8,8,8 \text { reps }) \\
\text { Week } 5(8,8,8 \text { reps }) \\
\text { Weeks } 6-9(8,8,8,8 \text { reps }\end{array}$ & $\begin{array}{l}\sim 75 \% 1 \mathrm{RM} \text { (adjusted to } \\
\text { maintain } 1-2 \mathrm{RIR} \text { ) }\end{array}$ \\
\hline
\end{tabular}

Exercise order is denoted by 1-3 (session one and three) and 1-2 (session two). Inter-set recovery periods were 120 seconds for HL-RT and 45 seconds for LL-BFR. Interexercise recovery periods for 3 minutes for both conditions. Prescribed tempo was $1 \mathrm{~s}$ for the concentric phase, and $1 \mathrm{~s}$ for the eccentric phase of repetitions, with no pause in between phases. PTBM, pre-training body mass; 1RM, one-repetition maximum; RIR, repetitions in reserve. 
Table 3. RT-qPCR primer details.

\begin{tabular}{lll}
\hline \multicolumn{1}{c}{ Target: } & \multicolumn{1}{c}{ Forward sequence: } & \multicolumn{1}{c}{ Reverse sequence: } \\
\hline GAPDH & AACCTGCCAAATATGATGAC & TCATACCAGGAAATGAGCTT \\
HPRT & TGACACTGGCAAAACAATGCA & GGTCCTTTCACCAGCAAGCT \\
$\mathbf{5 . 8 S}$ ribosomal RNA & ACTCTTAGCGGTGGATCACTC & GTGTCGATGATCAATGTGTCCTG \\
28S ribosomal RNA & TGACGCGATGTGATTTCTGC & TAGATGACGAGGCATTTGGC \\
$\mathbf{1 8 S}$ ribosomal RNA & TGCATGGCCGTTCTTAGTTG & AACGCCACTTGTCCCTCTAAG \\
$\mathbf{4 5 S}$ pre-ribosomal RNA & GCCTTCTCTAGCGATCTGAGAG & CCATAACGGAGGCAGAGACA \\
c-MYC & GGTAGTGGAAAACCAGCAGCC & TCTCCTCCTCGTCGCAGTA \\
POL1RA & CCTCAAGGTATCGCCCAGTC & GGCAACTTCTGTTCTTGGGC \\
TAF1A & AGGTTTAGCGCCTGCTCATA & CTGAAATCACTCATACCCGCCT \\
TIF1A & CATTTTGTGCCTCCCCGAGT & GTATTGGCATGAGAAACCACGG \\
UBF & AAGAAGCCTCCCATGAACGG & CGGCCAGCTTTTTGTAGTGC \\
\hline
\end{tabular}

Abbreviations: GAPDH, glyceraldehyde 3-phosphate dehydrogenase; HPRT, hypoxanthine guanine phosphoribosyl transferase; POL1RA, RNA polymerase 1 subunit A; TAF1A, TATA box-binding proteinassociated factor 1A; TIF1A, transcription initiation factor 1A; UBF, upstream binding transcription factor. 
Table 4. Assessments of muscular function measured before (Pre) and after (Post) training in the chronic study

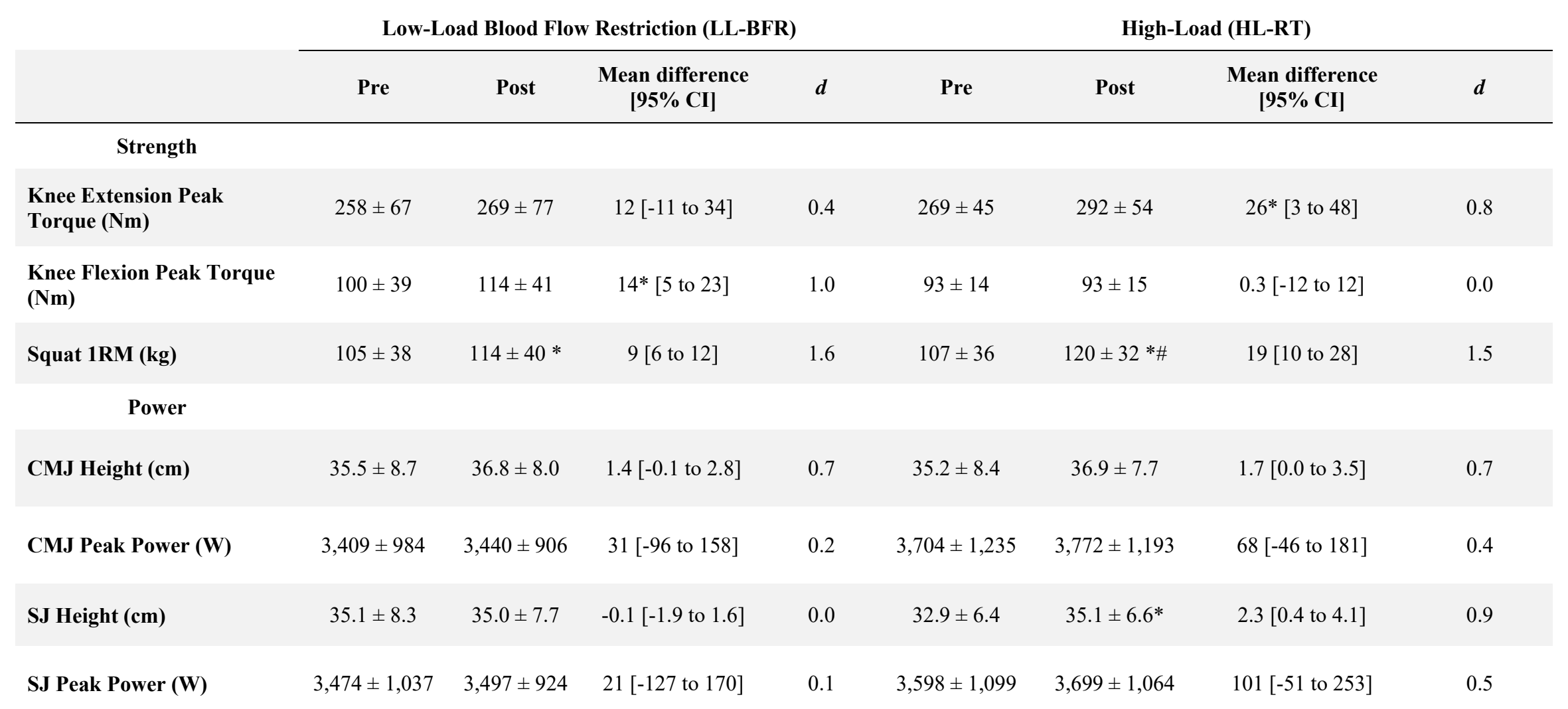

All data are presented as mean \pm SD values. $d$, Cohen's effect size. Nm, Newton-meter. 1RM, one-repetition maximum. CMJ, countermovement jump. SJ, squat jump. W, watts. *indicates a significant change from baseline $(p<0.05)$. \#indicates a significant difference from LL-BFR $(p<0.05)$. 


\section{Acute cellular and molecular responses and chronic adaptations to low-load blood flow restriction and high-load resistance exercise in trained individuals}

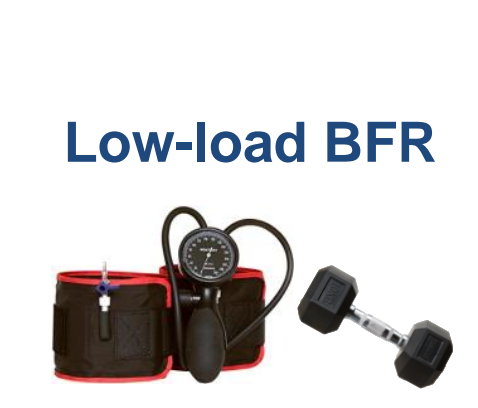

$30-40 \% 1 \mathrm{RM}$

VS

High-load RT

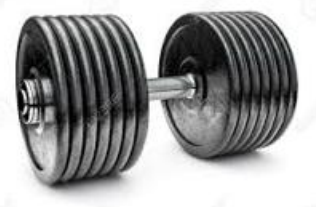

$75-80 \% 1 \mathrm{RM}$

\section{Chronic}

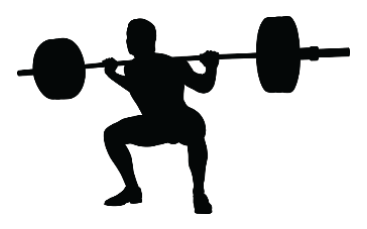

Squat $1 \mathrm{RM}$

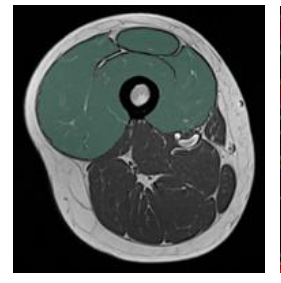

Acute
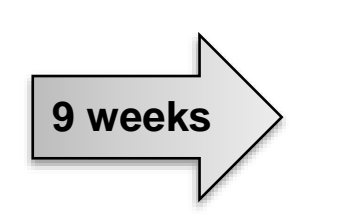
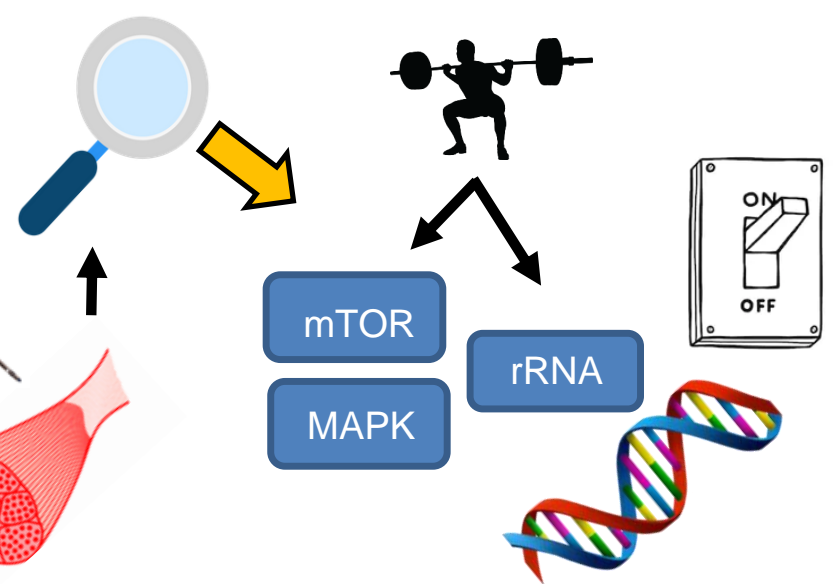

OUTCOMES

LL-BFR HL-RT

Quad CSA

Squat 1RM

Type II Fiber

Area

Type II Fiber

Myonuclei
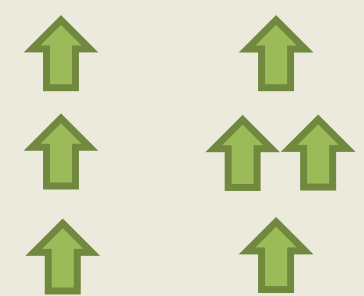

企

个

$\widehat{4}$

p70S6K

ERK1/2

C-Myc

TIF-1A

TAF-1A
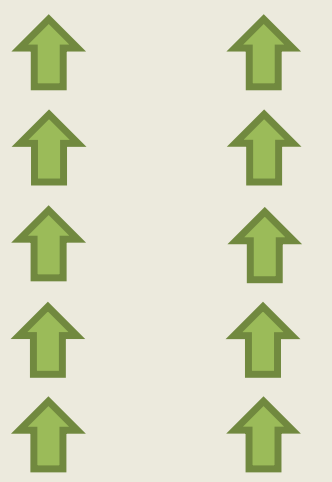

CONCLUSION Muscle mass, but not strength, increased similarly between training groups. Acute phosphorylation of key proteins involved in hypertrophy signaling pathways, and expression of ribosomal RNA transcription factors occurred to a similar degree with LL-BFR and HL-RT. Thus, low-load BFR is an effective

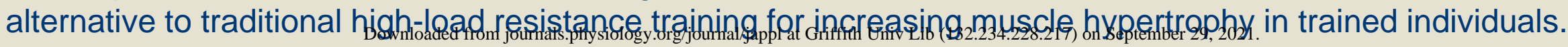


A

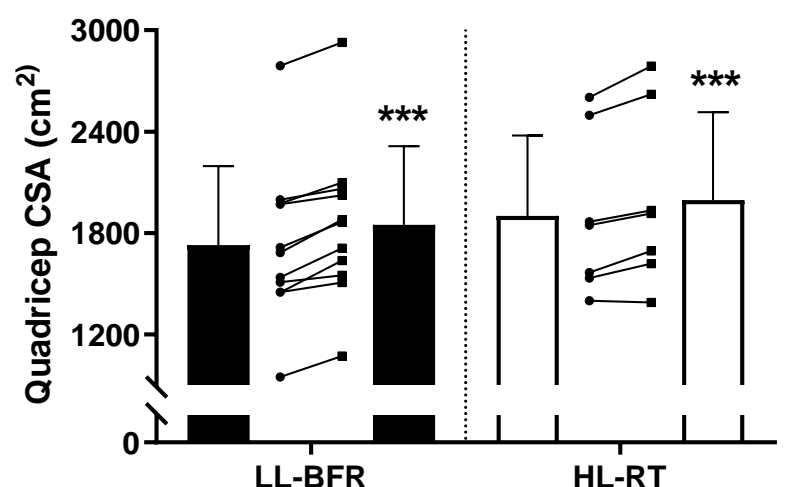

B

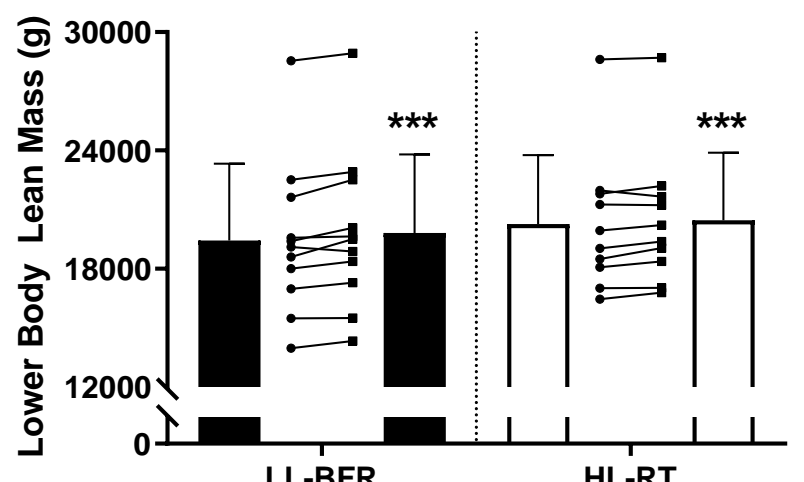

Downloaded from journals.physiology.org/journal/jappl at Griffith Univ Lib (132.234.228.217) on September 29, 2021. 
A

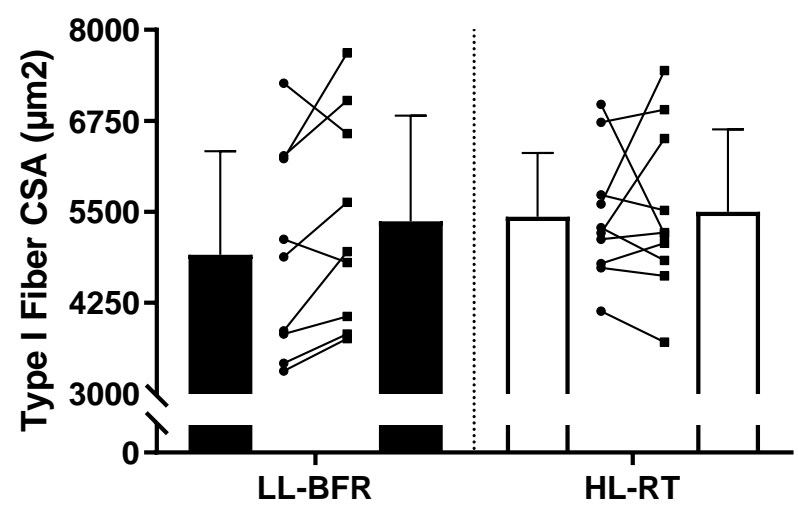

C

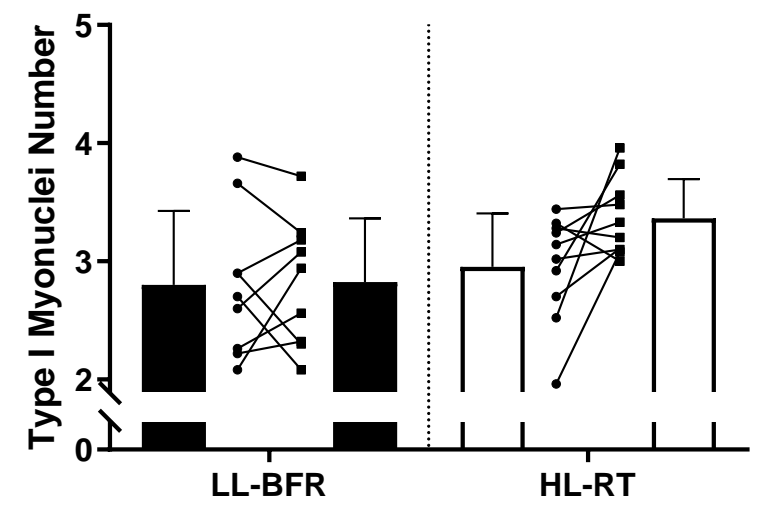

E

\section{B}

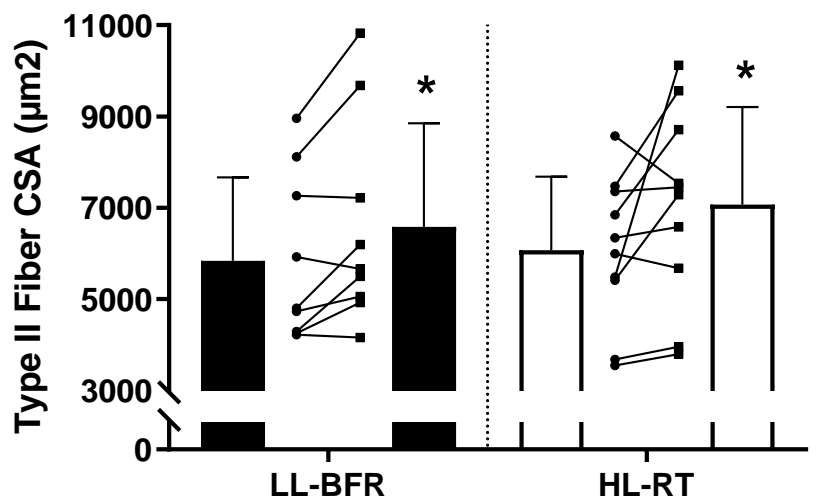

D

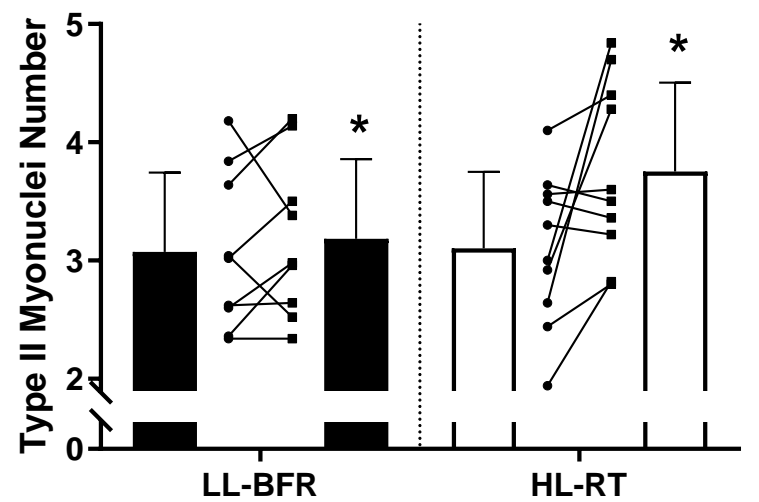

F

G 

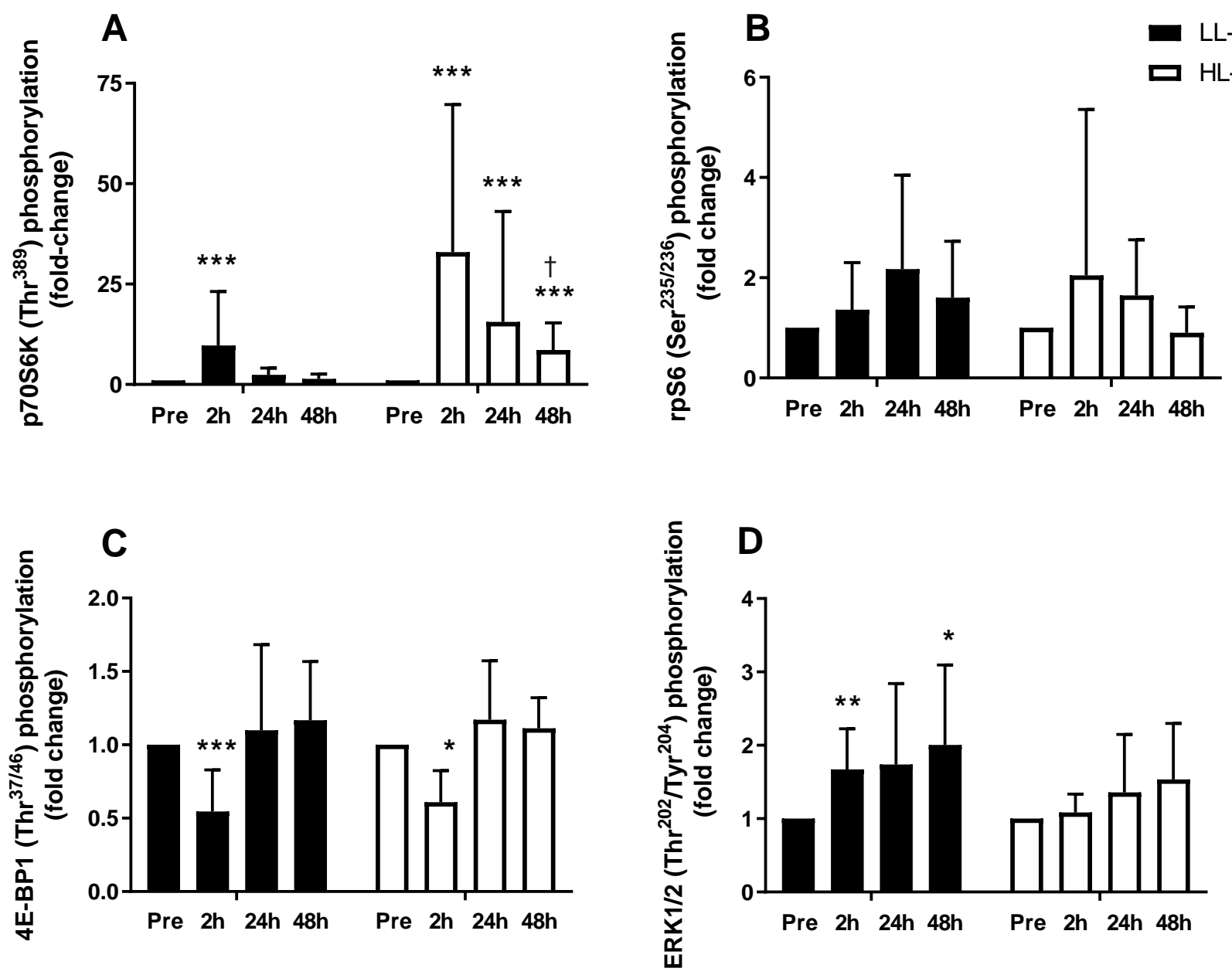

\section{E}

$70 \mathrm{kDa}-$
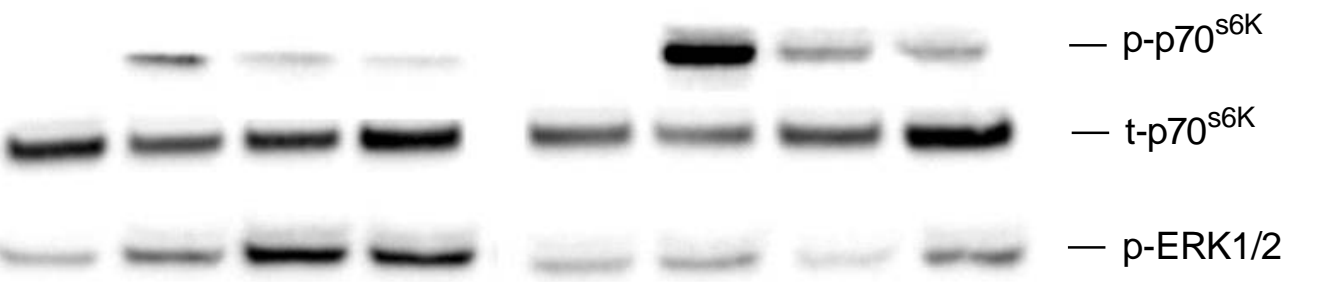

42-44 kDa -
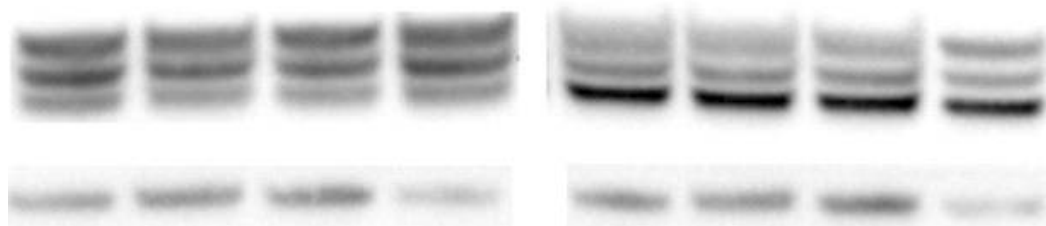

$32 \mathrm{kDa}-$

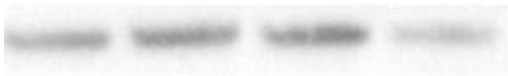

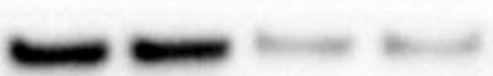

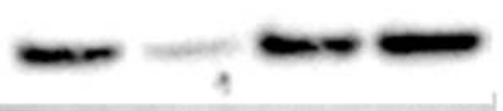

15-20 kDa -

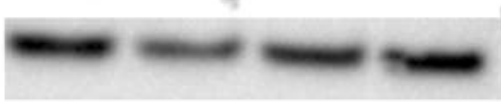

LL-BFR
- t-ERK1/2

- p-rpS6

- t-rpS6

- p-4EBP1

- t-4EBP1 
A

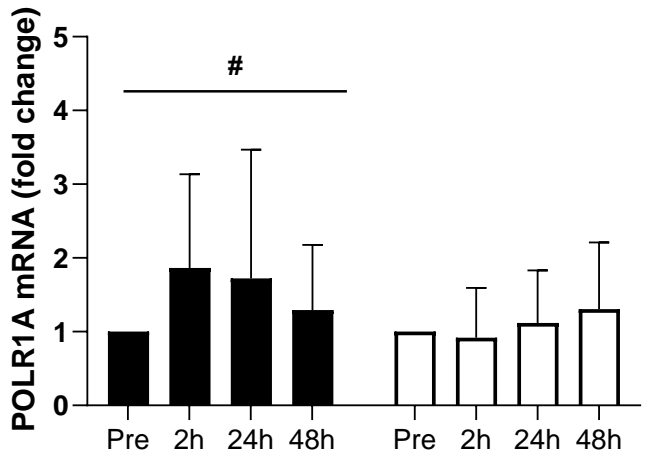

C

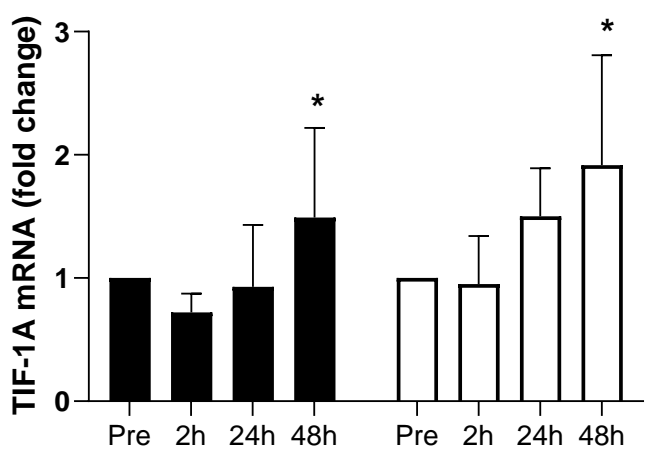

$E$

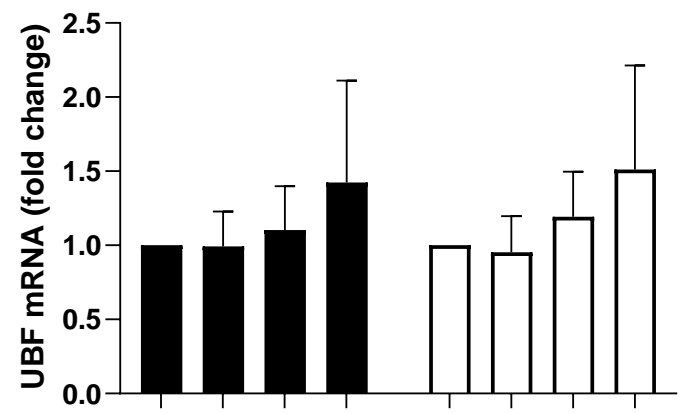

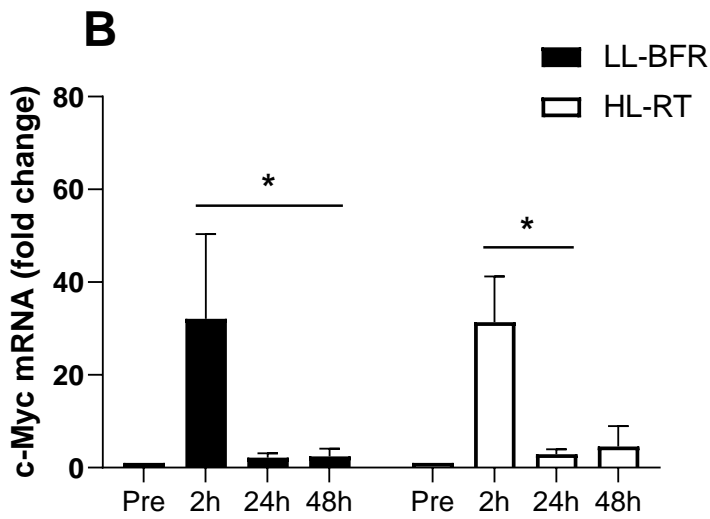
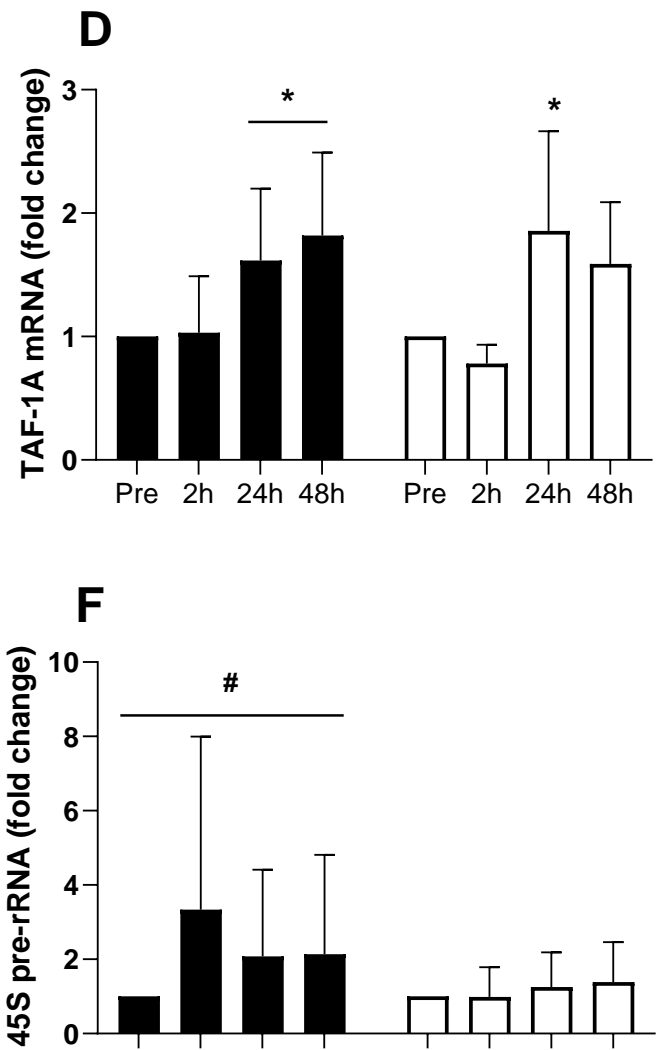

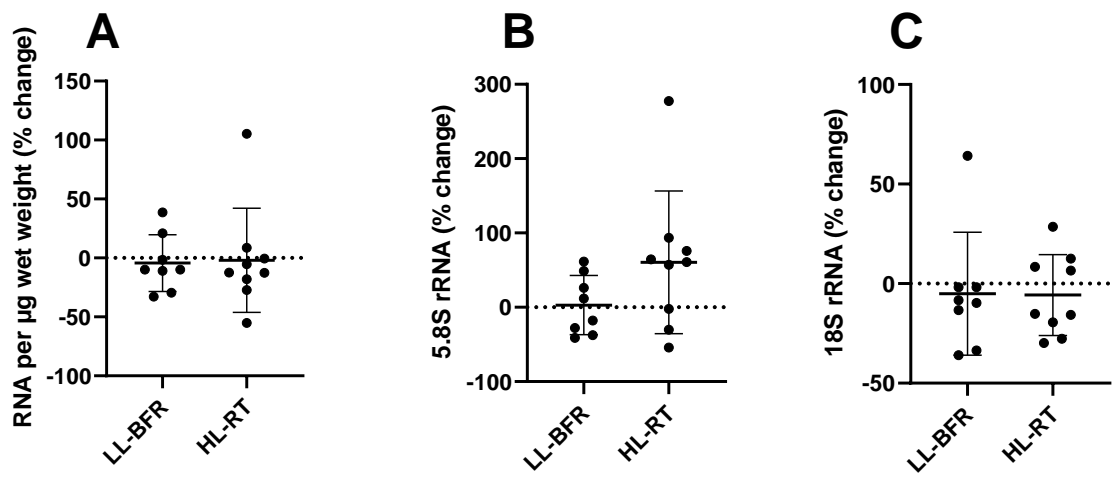

D

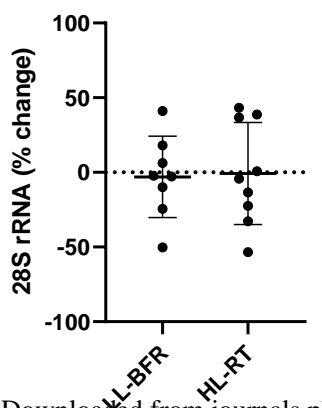

E

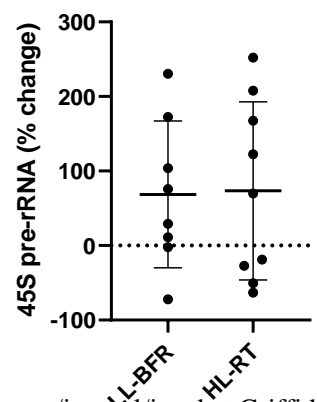

Downloađ̌ed from journals.physiology.org/jourral/jappl at Griffith Univ Lib (132.234.228.217) on September 29, 2021. 


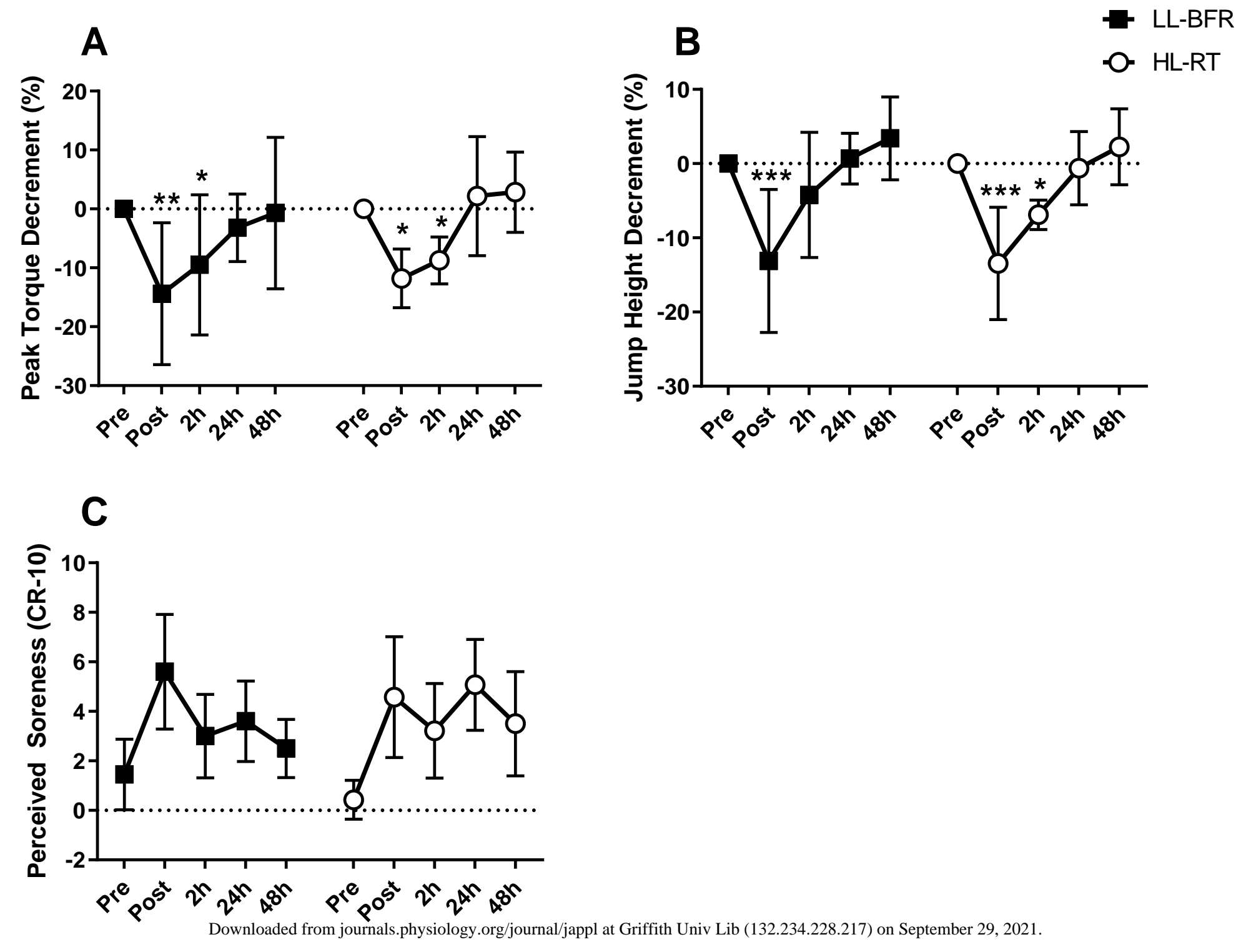

